\title{
含喹唑啉硫醚的杨梅素衍生物的设计、合成及生物活性研究
}

\author{
贺军唐雪梅 周清 彭 峰 刘婷婷 柳立伟 \\ 贺鸣谢承卫薛伟* \\ (贵州大学精细化工研究开发中心 绿色农药与农业生物工程国家重点实验室培育基地 \\ 教育部绿色农药与生物工程重点实验室 贵阳 550025)
}

\begin{abstract}
摘要 以杨梅苷为原料, 通过活性拼接, 设计并合成一系列含喹唑啉硫醚的杨梅素衍生物, 其结构通过 ${ }^{1} \mathrm{H}{ }^{\mathrm{N} M R} 、{ }^{13} \mathrm{C}$ NMR、 ${ }^{19} \mathrm{~F}$ NMR 和 HRMS 进行确证. 生物活性测试结果表明, 该类化合物对水稻白叶枯病菌 $(X$. Oryzae $)$ 、柑橘溃疡病 菌(X.Citri)和烟草青枯病菌( R. Solanacearum)表现出一定的抑制活性. 其中, 5,7-二甲氧基-3-(3-((6-溴喹唑啉-4-基)硫基) 丙氧基)-2-(3,4,5-三甲氧基苯基)-4H-色烯-4-酮( $\mathbf{A}_{15}$ )对水稻白叶枯病菌的 $\mathrm{EC}_{50}$ 值为 $13.9 \mu \mathrm{g} / \mathrm{mL}$, 优于对照药叶枯唑(88.9 $\mu \mathrm{g} / \mathrm{mL}$ )和噻菌铜 $(68.1 \mu \mathrm{g} / \mathrm{mL}) ; 5,7$-二甲氧基-3-(4-((6-氯喹唑啉-4-基)硫基)丁氧基)-2-(3,4,5-三甲氧基苯基)-4H-色烯-4-酮 (A) $\mathbf{A}_{3}$ 、5,7-二甲氧基-3-(3-((6-氯喹唑啉-4-基)硫基)丙氧基)-2-(3,4,5-三甲氧基苯基)-4H-色烯-4-酮( $\left.\mathbf{A}_{14}\right) 、 5,7$-二甲氧基3-(3-((6-溴喹唑啉-4-基)硫基)丙氧基)-2-(3,4,5-三甲氧基苯基)-4H-色烯-4-酮( $\mathbf{A}_{15}$ )和 5,7-二甲氧基-3-(3-((6-氟喹唑啉-4-基) 硫基)丙氧基)-2-(3,4,5-三甲氧基苯基)-4H-色烯-4-酮( $\mathbf{A}_{16}$ )对烟草青枯病菌的 $\mathrm{EC}_{50}$ 值分别为 1.1, 14.0, 11.9 和 $7.5 \mu \mathrm{g} / \mathrm{mL}$, 优于对照药叶枯唑(38.5 $\mu \mathrm{g} / \mathrm{mL})$ 和噻菌铜 $(184.8 \mu \mathrm{g} / \mathrm{mL})$. 活体实验结果表明, 化合物 $\mathbf{A}_{15}$ 对水稻白叶枯病菌的具有良好 治疗活性和保护活性. 通过扫描电镜成像初步探讨了目标化合物 $\mathbf{A}_{\mathbf{3}}$ 对烟草青枯病菌和 $\mathbf{A}_{15}$ 对水稻白叶枯病菌的抑菌作 用机制.
\end{abstract}

关键词 杨梅素衍生物; 喹唑啉硫醚; 生物活性; 活体实验; 扫描电镜

\section{Design, Synthesis and Biological Activities of Myricetin Derivatives Containing Quinazoline Thioether Moiety}
He, Jun
Tang, Xuemei
Zhou, Qing
Peng, Feng
Liu, Tingting

(Key Laboratory of Green Pesticide and Bioengineering of Ministry of Education, State Key Laboratory Breeding Base of Green Pesticide and Agricultural Bioengineering, Center for Research and Development of Fine Chemicals, Guizhou University, Guiyang 550025)

\begin{abstract}
A series of myricetin derivatives containing quinazoline thioether moiety were designed and synthesized using myricetrin as the starting material through active splicing strategy. All target compounds were characterized by ${ }^{1} \mathrm{H} N M R,{ }^{13} \mathrm{C}$ NMR, ${ }^{19} \mathrm{~F}$ NMR and HRMS. Their biological activities were evaluated. The results of biological activities showed that these compounds exhibited certain inhibitory activities against $X$. oryzae, $X$. citri and $R$. solanacearum. Among them, 3-(3-((6-bro-

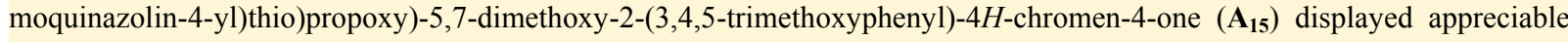
inhibitory effect against $X$. oryzae with a half maximal effective concentration $\left(\mathrm{EC}_{50}\right)$ values of $13.9 \mu \mathrm{g} / \mathrm{mL}$, which was better than those of the control drugs of bismerthiazol $(88.9 \mu \mathrm{g} / \mathrm{mL})$ and thiodiazole-copper $(68.1 \mu \mathrm{g} / \mathrm{mL})$. The EC Ev $_{50}$ values of 3-(4((6-chloroquinazolin-4-yl)thio)butoxy)-5,7-dimethoxy-2-(3,4,5-trimethoxyphenyl)-4H-chromen-4-one (A $)$, 3-(3-((6-chloro-

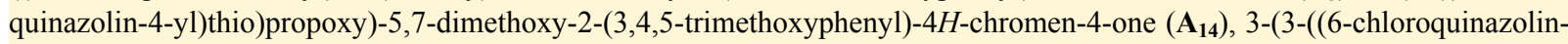
4-yl)thio)propoxy)-5,7-dimethoxy-2-(3,4,5-trimethoxyphenyl)-4H-chromen-4-one $\left(\mathbf{A}_{15}\right)$ and 3-(3-((6-fluoroquinazolin-4-yl)thio)propoxy)-5,7-dimethoxy-2-(3,4,5-trimethoxyphenyl)-4H-chromen-4-one ( $\left.\mathbf{A}_{\mathbf{1 6}}\right)$ against Ralstonia solanacearum were 1.1, 14.0, 11.9 and $7.5 \mu \mathrm{g} / \mathrm{mL}$, respectively, which were superior to the control drugs of bismerthiazol $(38.5 \mu \mathrm{g} / \mathrm{mL})$ and thio-
\end{abstract}

\footnotetext{
* Corresponding author. E-mail: wxue@gzu.edu.cn

Received August 7, 2020; revised September 4, 2020; published online October 10, 2020

Project supported by the National Natural Science Foundation of China (No. 21867003) and the Natural Science Foundation of Guizhou Province (Nos. 20192452, 20191105, 20171028).

国家自然科学基金(No. 21867003)和贵州省自然科学基金(Nos. 20192452, 20191105, 20171028)资助项目.
} 
diazole-copper $(184.8 \mu \mathrm{g} / \mathrm{mL})$. The results of in vivo experiments showed that compound $\mathbf{A}_{15}$ has good curative and protective activities against $X$. oryzae. The possible antibacterial mechanisms of target compound $\mathbf{A}_{\mathbf{3}}$ against $R$. solanacearum and $\mathbf{A}_{15}$ against $X$. oryzae were preliminarily discussed base on scanning electron microscope analyses.

Keywords myricetin derivative; quinazoline thioether; biological activity; in vivo experiment; scanning electron microscope

植物细菌性病害在农业生产过程中难以控制和管 理，每年都会对农业生产造成极大的损失 ${ }^{[1]}$. 目前市面 上出现了很多农用杀菌剂, 如叶枯唑和噻菌铜等. 随着 杀菌剂过度使用, 不仅部分植物病源菌对市面已有抗菌 剂产生了耐药性, 还会造成严重环境污染, 影响人类的 身体健康 ${ }^{[2]}$. 因此开发新的生物活性分子具有重要的意 义.

天然产物广泛存在于自然界, 具有毒性低、易降解、 结构新颖和对环境友好等特点 ${ }^{[3]}$, 在农药和医药的开发 过程具有重要的地位. 杨梅素是一种多羟基黄酮类化合

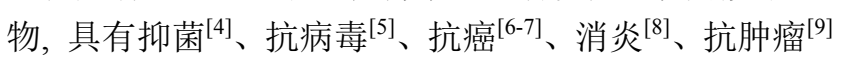
和抗氧化 ${ }^{[10]}$ 等多种生物活性. 本课题组在前期的工作 中, 发现了一系列具有抗菌活性的杨梅素衍生物. 蒋仕 春等 ${ }^{[11]}$ 将二硫代氨基甲酸酯结构引入到杨梅素结构中, 设计合成一系列新型杨梅素衍生物, 并对其进行生物活 性测试, 结果表明: 在浓度为 $100 \mu \mathrm{g} / \mathrm{mL}$ 时, 该系列化 合物对水稻白叶枯病菌和相橘溃疡病菌具有较好的抑 制活性. 李普等 ${ }^{[12]}$ 合成了一系列含喹喔啉的杨梅素衍 生物, 生物活性数据表明: 在 $100 \mu \mathrm{g} / \mathrm{mL}$ 浓度下, 该类 化合物对柑橘溃疡病菌均表现出了很好的抑制作用.

喹唑啉是一种具有芳香性的苯并嘧啶类含氮杂环 化合物, 具有多种生物活性, 如抗癌 ${ }^{[13-14]}$ 、抗菌 ${ }^{[15-16] 、}$ 抗病毒 ${ }^{[17]}$ 、抗增殖 ${ }^{[18]}$ 和杀虫 ${ }^{[19]}$ 等, 在农药和医药等领域 都有着广泛的应用 ${ }^{[20]}$. 李琴 ${ }^{[21]}$ 设计合成了一系列含喹 唑啉基团的 1,4-戊二烯-3-酮肜醚类化合物, 并对其进行 生物活性测试, 结果表明: 在 $100 \mu \mathrm{g} / \mathrm{mL}$ 浓度下, 大部 分化合物对柑橘溃疡病菌的抑制活性超过了对照药叶 枯唑. 刘军虎等 ${ }^{[2]}$ 设计并合成了一系列含 1,2,4-三唑席 夫碱的新型喹唑啉类化合物, 并对其进行抑菌活性测 试, 结果表明: 在 $50 \mu \mathrm{g} / \mathrm{mL}$ 浓度下, 部分目标化合物对 辣椒枯萎菌、苹果腐烂菌及马铃薯晚疫菌的表现了一定 的抑制活性. 同时, 在杂环化合物中引入硫原子可以增 加与受体的结合力, 有利于提高其生物活性 ${ }^{[23]}$, 硫醚结 构作为亚甲基的替代基团, 常常被用作农药创制中的有 效活性基团 ${ }^{[24]}$.

为了获得具有更好抑菌活性的杨梅素衍生物, 本工 作利用活性拼接原理, 结合课题组前期的研究基础, 将 4-硫酚喹唑啉引入到杨梅素结构中(Scheme 1), 合成了 22 个含喹唑啉硫醚的杨梅素衍生物, 采用浊度法测试 目标化合物的体外抑菌活性, 然后通过剪叶法测试化合 物 $\mathbf{A}_{15}$ 的对水稻白叶枯病菌的活体内的治疗活性和保护
活性, 最后通过扫描电镜成像初步探讨了目标化合物对 水稻白叶枯病菌和烟草青枯病菌可能的抑菌机制.

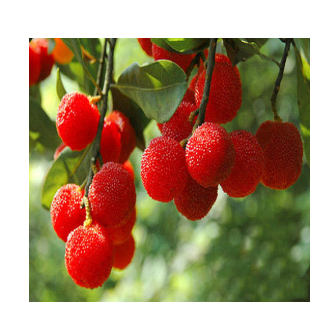<smiles>C=Cc1c(-c2cc(O)c(O)c(O)c2)oc2cc(O)cc(O)c2c1=O</smiles>
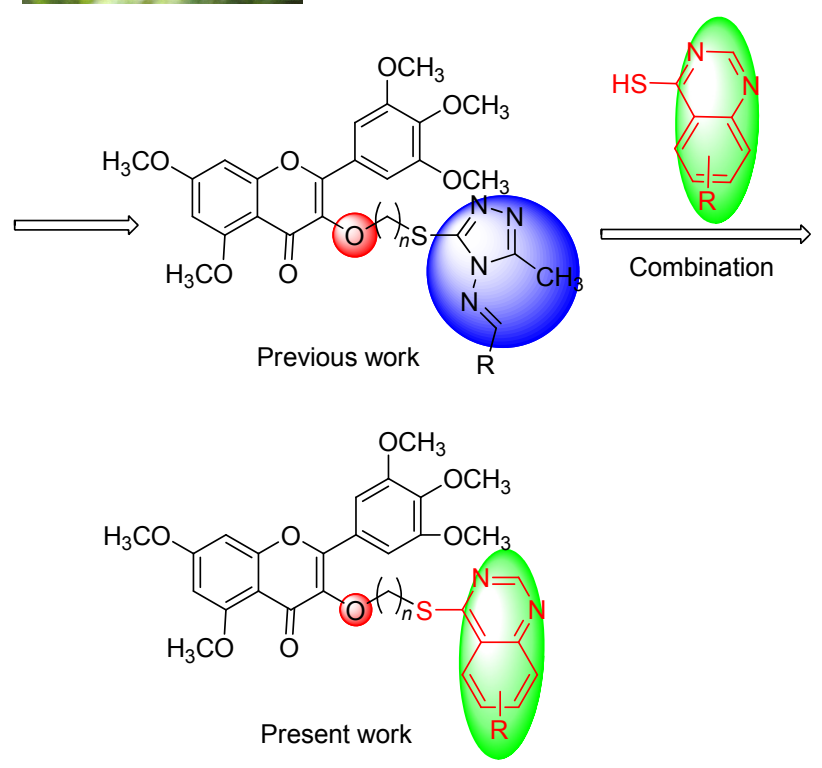

图式 1 目标化合物的设计思想

Scheme 1 Design strategy for target compounds

\section{1 结果与讨论}

\section{1 化合物的合成}

以杨梅苷为原料, 先与碘甲烷反应, 然后加入乙醇 和浓盐酸回流得到中间体 $\mathbf{1}$, 中间体 $\mathbf{1}$ 与二溴烷烃反应 生成中间体 2; 取代的邻氨基苯甲酸与甲酰胺在 $145{ }^{\circ} \mathrm{C}$ 关环制得中间体 3 , 再与二氯亚砜氯代生成中间体 4 , 进 一步与硫腿反应得到中间体 $\mathbf{5}$, 最后中间体 $\mathbf{5}$ 与中间体 $\mathbf{2}$ 发生取代反应得到目标化合物 $\mathbf{A}_{\mathbf{1}} \sim \mathbf{A}_{\mathbf{2 2}}$, 合成路线见 Scheme 2 .

\section{2 化合物的结构表征}

以目标化合物 $\mathbf{A}_{1}$ 为例, 对其 ${ }^{1} \mathrm{H} \mathrm{NMR} 、{ }^{13} \mathrm{C} N \mathrm{NR}$ 和 HRMS 的谱图进行讨论. 在 ${ }^{1} \mathrm{H}$ NMR 谱图中, 在 $\delta 8.89$ 出现单峰的是喹唑啉环上 2 位的氢, 在 $\delta 7.83$ 和 $\delta 7.66$ 出现峰的是喹唑啉环上 $5 、 7$ 和 8 位上的芳香氢, 在 $\delta$ 


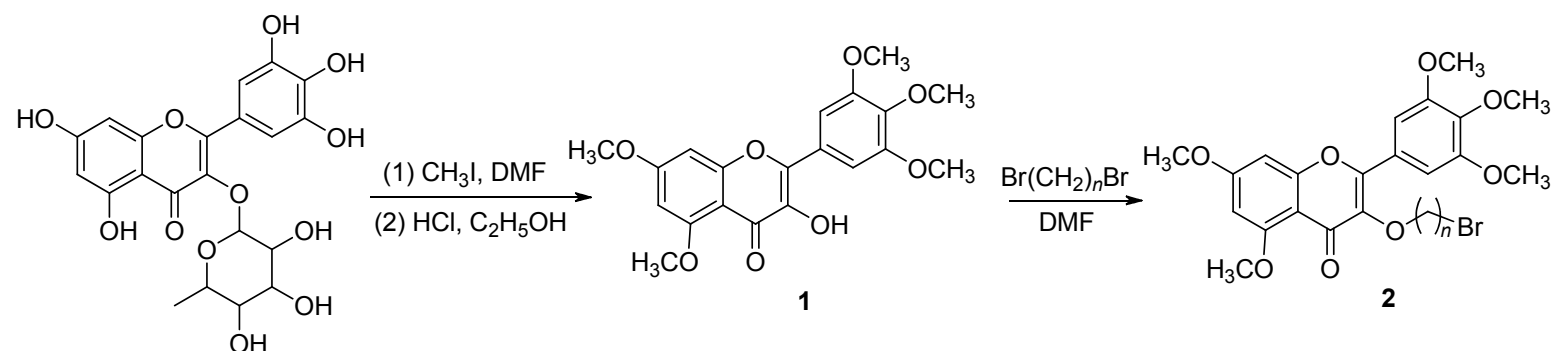

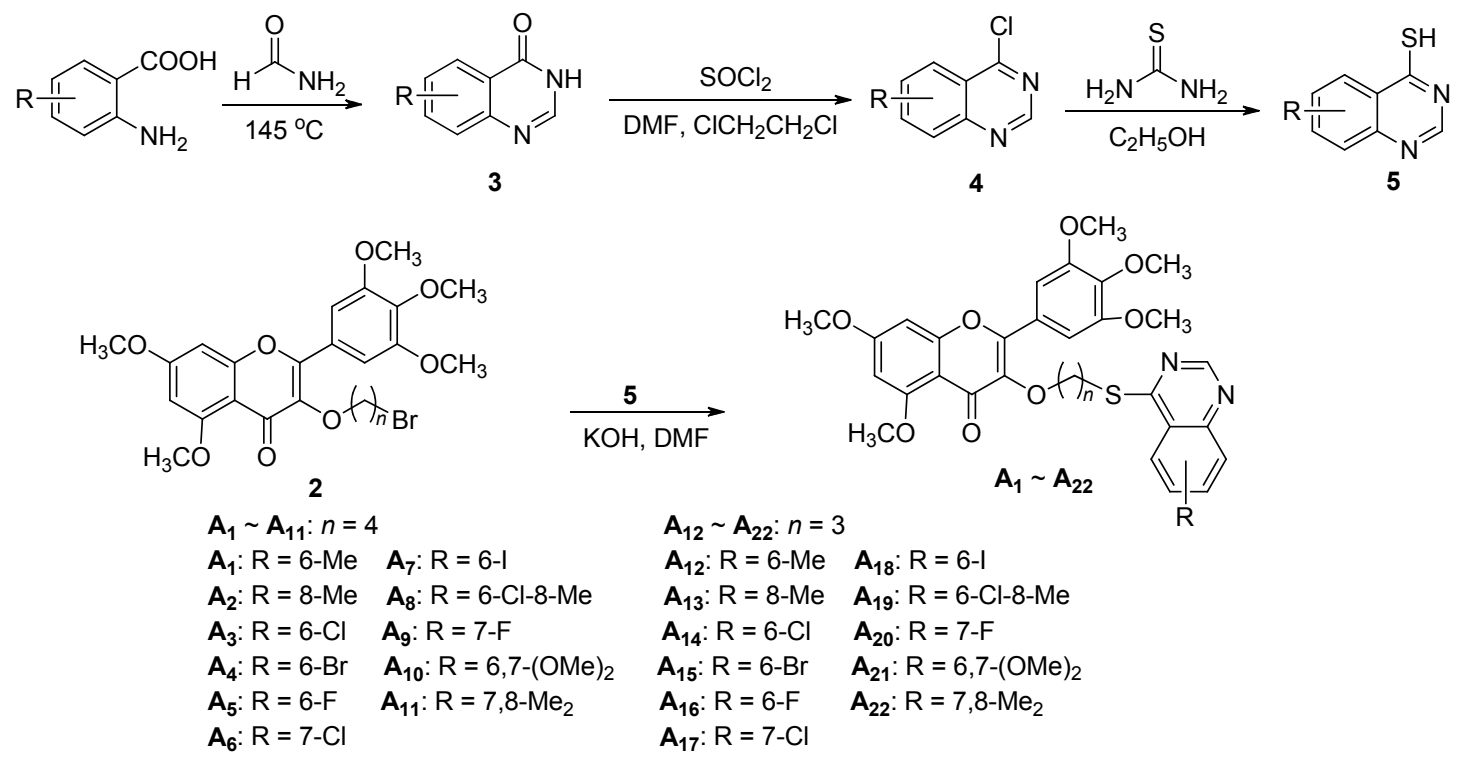

图式 2 目标化合物 $\mathbf{A}_{1} \sim \mathbf{A}_{22}$ 的合成路线

Scheme 2 Synthetic routes of target compounds $\mathbf{A}_{\mathbf{1}} \sim \mathbf{A}_{22}$

7.66、7.36 和 6.50 出现峰的是杨梅素上 4 个苯环上的芳 香氢, 在 $\delta 4.10$ 和 3.41 出现两个三重峰和 $\delta 1.99 \sim 1.91$ 出现峰的分别是含氧上亚甲基的氢、含硫上亚甲基的氢 和中间两个亚甲基的氢, 在 $\delta 3.97 \sim 3.88$ 出现峰的是杨 梅素上的五个苯甲氧基, 在 $\delta 2.55$ 出现单峰的是喹唑啉 环 6 位上的甲基. 在 ${ }^{13} \mathrm{C}$ NMR 谱图中, $\delta 174.02$ 和 21.80 处的峰为分子中 $\mathrm{C}=\mathrm{O}$ 和 $\mathrm{CH}_{3}$ 的碳原子吸收峰, $\delta 71.77$, $29.50,29.15$ 和 25.72 处的峰为四个亚甲基碳原子的吸收 峰. 在 HRMS 谱图中, 目标产物的 HRMS $[\mathrm{M}+\mathrm{H}]^{+}$实测 值和理论计算值是一致的, 误差均在 \pm 0.0030 范围内, 进一步证实了目标化合物的结构.

\section{3 生物活性测试}

\subsection{1 化合物的抑菌活性测试}

目标化合物 $\mathbf{A}_{\mathbf{1}} \sim \mathbf{A}_{22}$ 抑菌活性测试结果(表 1)表明, 大部分目标化合物对水稻白叶枯病菌、柑橘溃疡病菌和 烟草青枯病菌均有一定的抑制活性. 当浓度为 100 $\mu \mathrm{g} / \mathrm{mL}$ 时，化合物 $\mathbf{A}_{\mathbf{4}} 、 \mathbf{A}_{\mathbf{1 5}} 、 \mathbf{A}_{\mathbf{1 8}}$ 等对水稻白叶枯病菌的 抑制率超过对照药叶枯唑 (55.1\%)和噻菌铜 (62.2\%); 化 合物 $\mathbf{A}_{\mathbf{3}} \sim \mathbf{A}_{\mathbf{9}} 、 \mathbf{A}_{\mathbf{1 4}} \sim \mathbf{A}_{\mathbf{1 6}}$ 等对烟草青枯病菌的抑制率超过 对照药叶枯唑(59.5\%)和噻菌铜(38.4\%); 其中化合物 $\mathbf{A}_{15}$
对柑橘溃疡病菌、水稻白叶枯病菌和烟草青枯病菌的抑 菌率分别为 $58.0 \% 、 86.1 \%$ 和 $99.1 \%$, 超过对照药叶枯唑 $(51.4 \% 、 55.1 \%$ 和 59.5\%), 表现出较为广谱的抑菌活性.

\subsection{2 化合物的抑植物病菌 $\mathrm{EC}_{50}$ 值活性测试}

以商品药叶枯唑和噻菌铜为对照药, 测试了部分化 合物对水稻白叶枯病菌和烟草青枯病菌的 $\mathrm{EC}_{50}$ 值, 结 果列于表 2 和表 3. 由表 2 可知, 所测目标化合物 $\mathbf{A}_{1}$ 对 水稻白叶枯病菌的 $\mathrm{EC}_{50}$ 值为 $13.9 \mu \mathrm{g} / \mathrm{mL}$, 优于对照药叶 枯唑 $(88.9 \mu \mathrm{g} / \mathrm{mL})$ 和噻菌铜 $(68.1 \mu \mathrm{g} / \mathrm{mL})$. 由表 3 可知, 所测目标化合物对烟草青枯病菌 $\mathrm{EC}_{50}$ 值均优于对照药 噻菌铜 $(184.8 \mu \mathrm{g} / \mathrm{mL})$, 其中化合物 $\mathbf{A}_{\mathbf{3}} 、 \mathbf{A}_{\mathbf{1 4}} 、 \mathbf{A}_{\mathbf{1 5}}$ 和 $\mathbf{A}_{\mathbf{1 6}}$ 的 $\mathrm{EC}_{50}$ 值分别为 $1.1,14.0,11.9$ 和 $7.5 \mu \mathrm{g} / \mathrm{mL}$, 优于对照 药叶枯唑 $(38.5 \mu \mathrm{g} / \mathrm{mL})$.

根据表 1 3 数据可知, 对杨梅素进行部分甲基化 后，中间体 1 的抑菌活性优于杨梅素和杨梅苷，在引入 4-硫酚喹唑啉活性基团后，部分目标化合物表现出了较 好的抑菌活性. 初步构效关系分析表明：当喹唑啉环取 代基团为 6-Br 时，目标化合物对水稻白叶枯病菌均表 现出较好的抑制活性，如化合物 $\mathbf{A}_{\mathbf{4}}$ 和 $\mathbf{A}_{\mathbf{1 5}}$, 且化合物 $\mathbf{A}_{\mathbf{1 5}}$ 的 $\mathrm{EC}_{50}$ 值为 $13.9 \mu \mathrm{g} / \mathrm{mL}$, 优于对照药叶枯唑和噻菌铜; 
表 1 目标化合物 $\mathbf{A}_{\mathbf{1}} \sim \mathbf{A}_{22}$ 的抑菌活性(抑制率/\%) $)^{a}$

Table 1 Antibacterial activities (inhibition rate/\%) of target compounds $\mathbf{A}_{\mathbf{1}} \sim \mathbf{A}_{22}$

\begin{tabular}{|c|c|c|c|c|c|c|c|c|}
\hline \multirow{2}{*}{ Compd. } & \multirow{2}{*}{$\mathrm{R}$} & \multirow{2}{*}{$n$} & \multicolumn{2}{|c|}{$X$. oryzae } & \multicolumn{2}{|c|}{ X. citri } & \multicolumn{2}{|c|}{ R. solanacearum } \\
\hline & & & $100 \mu \mathrm{g} / \mathrm{mL}$ & $50 \mu \mathrm{g} / \mathrm{mL}$ & $100 \mu \mathrm{g} / \mathrm{mL}$ & $50 \mu \mathrm{g} / \mathrm{mL}$ & $100 \mu \mathrm{g} / \mathrm{mL}$ & $50 \mu \mathrm{g} / \mathrm{mL}$ \\
\hline $\mathbf{A}_{1}$ & 6-Me & 4 & $22.2 \pm 8.1$ & $20.0 \pm 4.2$ & $15.6 \pm 2.9$ & $14.9 \pm 1.3$ & - & - \\
\hline $\mathbf{A}_{2}$ & 8-Me & 4 & $32.7 \pm 0.1$ & $16.6 \pm 3.5$ & $10.1 \pm 5.3$ & $6.7 \pm 2.9$ & $21.5 \pm 1.6$ & $13.3 \pm 0.5$ \\
\hline $\mathbf{A}_{3}$ & $\mathrm{Cl}$ & 4 & $59.9 \pm 4.6$ & $27.3 \pm 0.3$ & $11.3 \pm 2.7$ & - & $88.4 \pm 5.5$ & $83.8 \pm 4.4$ \\
\hline $\mathbf{A}_{4}$ & $6-\mathrm{Br}$ & 4 & $64.2 \pm 5.2$ & $42.0 \pm 0.6$ & - & - & $86.8 \pm 3.5$ & $55.5 \pm 4.5$ \\
\hline $\mathbf{A}_{5}$ & $6-\mathrm{F}$ & 4 & $42.2 \pm 6.4$ & $35.5 \pm 1.1$ & $11.2 \pm 3.8$ & $9.6 \pm 1.9$ & $66.9 \pm 3.8$ & $44.1 \pm 2.4$ \\
\hline $\mathbf{A}_{6}$ & 7-Cl & 4 & $30.9 \pm 4.9$ & $28.4 \pm 5.2$ & $8.5 \pm 3.4$ & $6.7 \pm 1.0$ & $65.8 \pm 3.4$ & $57.1 \pm 3.9$ \\
\hline $\mathbf{A}_{7}$ & $6-\mathrm{I}$ & 4 & $28.7 \pm 3.9$ & $26.5 \pm 2.6$ & - & - & $80.7 \pm 2.9$ & $63.0 \pm 3.5$ \\
\hline $\mathbf{A}_{8}$ & 6-Cl-8-Me & 4 & $42.6 \pm 3.4$ & $36.6 \pm 0.9$ & $30.0 \pm 5.0$ & $23.3 \pm 1.7$ & $70.4 \pm 1.3$ & $77.3 \pm 6.8$ \\
\hline $\mathbf{A}_{9}$ & $7-\mathrm{F}$ & 4 & $50.4 \pm 2.0$ & $30.1 \pm 1.3$ & $16.6 \pm 0.8$ & $22.2 \pm 3.4$ & $70.8 \pm 4.0$ & $59.1 \pm 3.9$ \\
\hline $\mathbf{A}_{10}$ & $6,7-(\mathrm{OMe})_{2}$ & 4 & $41.4 \pm 2.4$ & $28.1 \pm 2.0$ & $8.8 \pm 2.2$ & $5.3 \pm 1.6$ & $40.7 \pm 6.1$ & $27.1 \pm 7.0$ \\
\hline $\mathbf{A}_{11}$ & $7,8-\mathrm{Me}_{2}$ & 4 & $40.4 \pm 4.4$ & $34.2 \pm 5.5$ & - & - & $22.2 \pm 5.4$ & $16.9 \pm 6.5$ \\
\hline $\mathbf{A}_{12}$ & 6-Me & 3 & $23.7 \pm 4.5$ & $23.4 \pm 1.7$ & $12.5 \pm 1.2$ & $12.7 \pm 2.3$ & $12.1 \pm 5.4$ & - \\
\hline $\mathbf{A}_{13}$ & 8-Me & 3 & $26.8 \pm 2.8$ & $23.2 \pm 7.4$ & $11.5 \pm 2.9$ & $4.4 \pm 1.8$ & $8.7 \pm 4.3$ & - \\
\hline $\mathbf{A}_{14}$ & $6-\mathrm{Cl}$ & 3 & $44.6 \pm 2.5$ & $29.8 \pm 2.9$ & $33.1 \pm 2.2$ & $13.3 \pm 2.2$ & $77.6 \pm 1.3$ & $71.3 \pm 3.8$ \\
\hline $\mathbf{A}_{15}$ & $6-\mathrm{Br}$ & 3 & $86.1 \pm 5.1$ & $71.4 \pm 4.5$ & $58.0 \pm 2.3$ & $12.1 \pm 1.1$ & $99.1 \pm 3.0$ & $76.2 \pm 3.6$ \\
\hline$A_{16}$ & $6-\mathrm{F}$ & 3 & $44.9 \pm 3.2$ & $40.6 \pm 4.7$ & - & - & $73.1 \pm 2.7$ & $66.0 \pm 5.2$ \\
\hline $\mathbf{A}_{17}$ & 7-Cl & 3 & $31.2 \pm 2.8$ & $28.4 \pm 3.2$ & $19.3 \pm 1.2$ & $15.2 \pm 0.6$ & $19.7 \pm 3.5$ & $12.7 \pm 1.6$ \\
\hline $\mathbf{A}_{18}$ & $6-\mathrm{I}$ & 3 & $62.8 \pm 3.8$ & $39.9 \pm 5.6$ & $56.2 \pm 3.4$ & $28.3 \pm 0.9$ & $35.1 \pm 5.2$ & $19.1 \pm 1.2$ \\
\hline$A_{19}$ & 6-Cl-8-Me & 3 & $42.7 \pm 3.4$ & $36.0 \pm 7.4$ & $21.1 \pm 5.0$ & $19.0 \pm 2.2$ & $50.8 \pm 1.6$ & $42.8 \pm 0.7$ \\
\hline $\mathbf{A}_{20}$ & $7-\mathrm{F}$ & 3 & $41.4 \pm 4.4$ & $29.2 \pm 0.8$ & $23.8 \pm 1.6$ & $10.6 \pm 4.2$ & $63.3 \pm 8.7$ & $39.1 \pm 4.5$ \\
\hline $\mathbf{A}_{21}$ & $6,7-(\mathrm{OMe})_{2}$ & 3 & $34.1 \pm 2.8$ & $24.5 \pm 0.8$ & $12.1 \pm 3.0$ & - & $12.1 \pm 4.1$ & $6.6 \pm 2.9$ \\
\hline $\mathbf{A}_{22}$ & $7,8-\mathrm{Me}_{2}$ & 3 & $58.0 \pm 2.3$ & $47.7 \pm 0.4$ & $18.3 \pm 5.3$ & $11.6 \pm 6.9$ & $38.5 \pm 2.9$ & $33.4 \pm 6.7$ \\
\hline 中间体 $\mathbf{1}$ & - & - & $35.5 \pm 1.6$ & $18.3 \pm 1.9$ & $18.9 \pm 4.0$ & $9.3 \pm 2.8$ & $40.8 \pm 2.0$ & $14.0 \pm 1.3$ \\
\hline 杨梅素 & - & - & $30.3 \pm 5.3$ & $10.6 \pm 0.5$ & $11.0 \pm 3.5$ & - & $9.3 \pm 4.5$ & $5.2 \pm 3.0$ \\
\hline 杨梅苷 & - & - & $10.9 \pm 3.9$ & $8.9 \pm 5.1$ & - & - & $20.9 \pm 2.0$ & $7.7 \pm 4.8$ \\
\hline 叶枯唑 ${ }^{b}$ & - & - & $55.1 \pm 1.82$ & $39.0 \pm 1.8$ & $51.4 \pm 2.0$ & $19.2 \pm 2.0$ & $59.5 \pm 5.7$ & $51.7 \pm 0.2$ \\
\hline 噻菌铜 ${ }^{b}$ & - & - & $62.2 \pm 4.8$ & $36.1 \pm 4.4$ & $63.3 \pm 0.6$ & $18.5 \pm 6.0$ & $38.4 \pm 3.8$ & $20.9 \pm 1.6$ \\
\hline
\end{tabular}

${ }^{a}$ Average of three replicates. ${ }^{b}$ The commercial bactericides (bismerthiazol and thiodiazole-copper) were used for comparison of antibacterial activity.

表 2 部分目标化合物对水稻白叶枯病菌的 $\mathrm{EC}_{50}$ 值

Table $2 \mathrm{EC}_{50}$ values of some compounds against $X$. oryzae

\begin{tabular}{cccccc}
\hline Compd. & $n$ & $\mathrm{R}$ & Toxic regression equation & $r$ & $\mathrm{EC}_{50} /\left(\mu \mathrm{g} \cdot \mathrm{mL}^{-1}\right)$ \\
\hline $\mathbf{A}_{\mathbf{4}}$ & 4 & $6-\mathrm{Br}$ & $y=1.8096 x+1.6782$ & 0.9627 & 68.5 \\
$\mathbf{A}_{\mathbf{1 5}}$ & 3 & $6-\mathrm{Br}$ & $y=1.1947 x+3.6346$ & 0.9934 & 13.9 \\
$\mathbf{A}_{\mathbf{1 8}}$ & 3 & $6-\mathrm{I}$ & $y=1.3339 x+2.4922$ & 0.9715 & 75.9 \\
噻菌铜 $^{a}$ & - & - & $y=1.8570 x+1.5964$ & 0.9910 & 68.1 \\
叶枯唑 $^{a}$ & - & - & $y=1.2079 x+2.6426$ & 0.9844 & 88.9 \\
\hline
\end{tabular}

${ }^{a}$ The commercial bactericides (bismerthiazol and thiodiazole-copper) were used for comparison of antibacterial activity.

表 3 部分目标化合物对烟草青枯病菌的 $\mathrm{EC}_{50}$ 值

Table $3 \quad \mathrm{EC}_{50}$ values of some compounds against $R$. solanacearum

\begin{tabular}{cllccc}
\hline Compd. & $n$ & \multicolumn{1}{c}{$\mathrm{R}$} & Toxic regression equation & $r$ & $\mathrm{EC}_{50} /\left(\mu \mathrm{g} \cdot \mathrm{mL}^{-1}\right)$ \\
\hline $\mathbf{A}_{\mathbf{3}}$ & 4 & 6-Cl & $y=0.593 x+4.9827$ & 0.9921 & 1.1 \\
$\mathbf{A}_{\mathbf{4}}$ & 4 & 6-Br & $y=1.8966 x+2.1798$ & 0.9632 & 30.7 \\
$\mathbf{A}_{\mathbf{5}}$ & 4 & 6-F & $y=0.8597 x+3.5656$ & 0.9585 & 46.6 \\
$\mathbf{A}_{\mathbf{6}}$ & 4 & 7-Cl & $y=0.5752 x+4.2419$ & 0.9636 & 20.1 \\
$\mathbf{A}_{\mathbf{7}}$ & 4 & 6-I & $y=1.4886 x+2.9695$ & 0.9507 & 23.1 \\
$\mathbf{A}_{\mathbf{8}}$ & 4 & 6-Cl-8-Me & $y=1.4015 x+3.064$ & 0.9633 & 24.1 \\
$\mathbf{A}_{\mathbf{9}}$ & 4 & 7-F & $y=1.3577 x+2.8057$ & 0.9934 & 41.3 \\
$\mathbf{A}_{\mathbf{1 4}}$ & 3 & 6-Cl & $y=0.9359 x+3.9275$ & 0.9617 & 14.0 \\
$\mathbf{A}_{\mathbf{1 5}}$ & 3 & 6- $\mathrm{Br}$ & $y=1.7487 x+3.0242$ & 0.9766 & 11.9 \\
$\mathbf{A}_{\mathbf{1 6}}$ & 3 & 6-F & $y=0.8035 x+4.2979$ & 0.9904 & 7.5 \\
$\mathbf{A}_{\mathbf{2 0}}$ & 3 & 6-Cl-8-Me & $y=0.538 x+3.9015$ & 0.9582 & 110.1 \\
\hline
\end{tabular}




\begin{tabular}{cccccc}
\hline Compd. & $n$ & $\mathrm{R}$ & Toxic regression equation & $r$ & $\mathrm{EC}_{50} /\left(\mu \mathrm{g} \cdot \mathrm{mL}^{-1}\right)$ \\
\hline 噻菌铜 $^{a}$ & - & - & $y=0.6931 x+3.4289$ & 0.9773 & 184.8 \\
叶枯唑 $^{a}$ & - & - & $y=0.8701 x+4.0372$ & 0.9753 & 38.5 \\
\hline
\end{tabular}

${ }^{a}$ The commercial bactericides (bismerthiazol and thiodiazole-copper) were used for comparison of antibacterial activity.

当喹唑啉环上取代基团含有卤素时, 目标化合物对烟草 青枯病菌表现出较好的抑制活性, 如 $\mathbf{A}_{\mathbf{3}} 、 \mathbf{A}_{\mathbf{1 4}} 、 \mathbf{A}_{\mathbf{1 5}}$ 和 $\mathbf{A}_{16}$ 的 $\mathrm{EC}_{50}$ 值分别为 $1.1,14.0,11.9$ 和 $7.5 \mu \mathrm{g} / \mathrm{mL}$, 优于 对照药剂叶枯唑和噻菌铜. 但是, 当取代基为甲基 $\left(\mathbf{A}_{1}, \mathbf{A}_{2}, \mathbf{A}_{11}, \mathbf{A}_{12}, \mathbf{A}_{13}\right.$ 和 $\left.\mathbf{A}_{22}\right)$ 或甲氧基 $\left(\mathbf{A}_{10}\right.$ 和 $\left.\mathbf{A}_{21}\right)$ 时，目标 化合物的抑菌活性比较差. 综上所述, 当喹唑啉环上取 代基含有卤素时, 目标化合物的抑菌活性将会得到提 升.

1.3.3 目标化合物 $\mathbf{A}_{15}$ 抗水稻白叶枯病菌 $(X$. oryzae $)$ 活体测试

化合物 $\mathbf{A}_{15}$ 具有良好的体外抑菌活性, 通过剪叶法 进一步探讨了它们对水稻白叶枯病的体内抑制作用. 以 叶枯唑和噻菌铜铜作为阳性对照. $16 \mathrm{~d}$ 后所有接种的水 稻植株均表现出枯萎病症状，发病率为 $100 \%$ 。

从表 4 可知，在浓度为 $200 \mu \mathrm{g} / \mathrm{mL}$ 时，化合物 $\mathbf{A}_{15}$ 的治疗活性的控制效率为 $49.4 \%$, 优于对照药剂叶枯唑 (45.3\%)和噻菌铜(34.8\%). 从表 5 可知, 化合物 $\mathbf{A}_{15}$ 的保 护活性的控制效率为 $47.2 \%$, 优于对照药剂噻菌铜 $35.1 \%$, 且接近于对照药剂叶枯唑 $47.7 \%$.

表 4 化合物 $\mathbf{A}_{15}$ 对水稻白叶枯病菌的治疗作用 Table 4 Curative effect of compound $\mathbf{A}_{15}$ against $X$. oryzae

\begin{tabular}{lccc}
\hline Treatment & $\begin{array}{c}\text { Morbidity/ } \\
\%\end{array}$ & $\begin{array}{c}\text { Disease index/ } \\
\%\end{array}$ & $\begin{array}{c}\text { Control efficiency/ } \\
\%\end{array}$ \\
\hline $\mathbf{A}_{15}$ & 100 & 44.1 & 49.4 \\
叶枯唑 $^{a}$ & 100 & 47.6 & 45.3 \\
噻菌铜 $^{a}$ & 100 & 56.8 & 34.8 \\
$\mathrm{CK}^{b}$ & 100 & 87.0 & - \\
\hline
\end{tabular}

${ }^{a}$ The commercial bactericides (bismerthiazol and thiodiazole-copper) were used for positive controls. ${ }^{b}$ Negative control.

表 5 化合物 $\mathbf{A}_{15}$ 对水稻白叶枯病菌的保护作用 Table 5 Protective effect of compound $\mathbf{A}_{15}$ against $X$. oryzae.

\begin{tabular}{lccc}
\hline Treatment & $\begin{array}{c}\text { Morbidity/ } \\
\%\end{array}$ & $\begin{array}{c}\text { Disease index/ } \\
\%\end{array}$ & $\begin{array}{c}\text { Control efficiency/ } \\
\%\end{array}$ \\
\hline $\mathbf{A}_{15}$ & 100 & 45.9 & 47.2 \\
叶枯唑 $^{a}$ & 100 & 45.5 & 47.7 \\
噻菌铜 $^{a}$ & 100 & 56.5 & 35.1 \\
$\mathrm{CK}^{b}$ & 100 & 87.0 & - \\
\hline
\end{tabular}

${ }^{a}$ The commercial bactericides (bismerthiazol and thiodiazole-copper) were used for positive controls. ${ }^{b}$ Negative control.

1.3.4 目标化合物 $\mathbf{A}_{3}$ 抑烟草青枯病菌 $(R$. solanacearum) 和 $\mathbf{A}_{15}$ 抑水稻白叶枯病菌 $(X$. oryzae)作用机制初 步研究

根据扫描电镜观察烟草青枯病菌( $R$. solanacearum)
和水稻白叶枯病菌 $(X$. oryzae) 表面形态(图 3,4), 发现烟 草青枯病菌 $(R$. solanacearum $)$ 的空白对照组菌体呈短粗 的杆状形状，表面光滑且大小均一，形貌完整；当用 $50 \mu \mathrm{g} / \mathrm{mL}$ 的 $\mathbf{A}_{3}$ 药液处理后部分菌体表面出现褶皱; 当 用 $100 \mu \mathrm{g} / \mathrm{mL}$ 的 $\mathrm{A}_{3}$ 药液处理后菌体禇皱更为明显，部分 菌体两端凹陷. 水稻白叶枯病菌 $(X$. oryzae $)$ 的空白对照 组菌体呈细长的杆状形状结构且形貌结构完整; 当用 $50 \mu \mathrm{g} / \mathrm{mL}$ 的 $\mathbf{A}_{15}$ 药液处理后部分菌体表面发生凹陷; 当 用 $100 \mu \mathrm{g} / \mathrm{mL}$ 的 $\mathbf{A}_{15}$ 药液处理后大量的菌体出现干瘜和 卷曲. 扫描电镜成像进一步验证了化合物的抑菌活性.
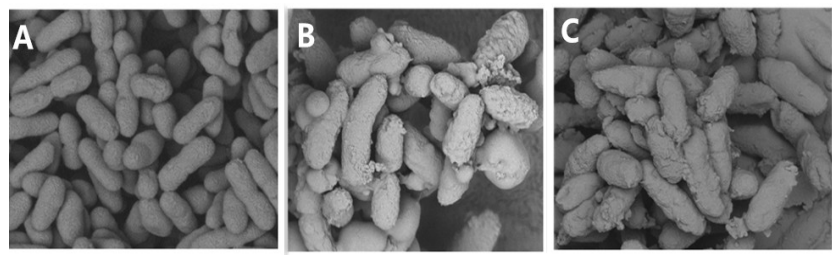

图 3 不同浓度 $\mathbf{A}_{\mathbf{3}}$ 作用下的烟草青枯病菌扫描电镜图像 Figure 3 SEM images for $R$. solanacearum after incubated in different concentration of compound $\mathbf{A}_{3}$

(A) $0 \mu \mathrm{g} / \mathrm{mL}$, (B) $50 \mu \mathrm{g} / \mathrm{mL}$, (C) $100 \mu \mathrm{g} / \mathrm{mL}$
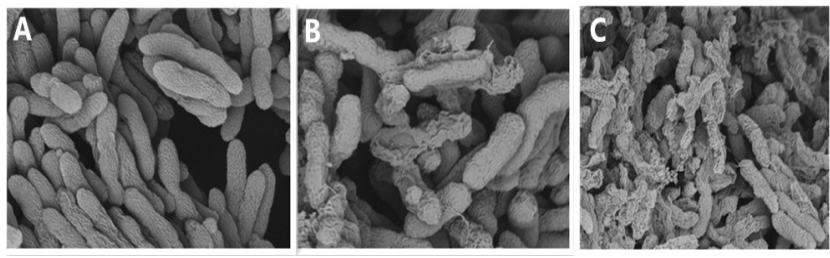

图4 不同浓度 $\mathbf{A}_{15}$ 作用下的水稻白叶枯病菌扫描电镜图像

Figure 4 SEM images for $X$. oryzae after incubated in different concentration of compound $\mathbf{A}_{15}$

(A) $0 \mu \mathrm{g} / \mathrm{mL}$, (B) $50 \mu \mathrm{g} / \mathrm{mL},(C) 100 \mu \mathrm{g} / \mathrm{mL}$

\section{2 结论}

以杨梅苷、取代的邻氨基苯甲酸、1,3-二溴丙烷和 $1,4-$ 二溴丁烷等为原料, 经多步反应合成了 22 个新型含 喹唑啉硫醚的杨梅素衍生物. 初步抑菌活性测试结果表 明: 在引入了 4-硫酚喹唑啉活性片段后, 大多数目标化 合物对柑橘溃疡病菌、水稻白叶枯病菌和烟草青枯病菌 都有一定抑制活性. 部分化合物如 $\mathbf{A}_{\mathbf{3}}, \mathbf{A}_{\mathbf{1 4}}, \mathbf{A}_{\mathbf{1 5}}$ 和 $\mathbf{A}_{\mathbf{1 6}}$ 对 烟草青枯病菌的 $\mathrm{EC}_{50}$ 值分别为 $1.1,14.0,11.9$ 和 7.5 $\mu \mathrm{g} / \mathrm{mL}$, 优于对照药叶枯唑 $(38.5 \mu \mathrm{g} / \mathrm{mL}$ ) 和噻菌铜 $(184.4$ $\mu \mathrm{g} / \mathrm{mL}$ ). 其中目标化合物 $\mathbf{A}_{15}$ 对水稻白叶枯病菌和烟草 青枯病菌的 $\mathrm{EC}_{50}$ 值分别为 13.9 和 $11.9 \mu \mathrm{g} / \mathrm{mL}$, 均优于对 照药叶枯唑和噻菌铜, 且浓度为 $100 \mu \mathrm{g} / \mathrm{mL}$ 时, 对柑橘 
溃疡病菌的抑制活性也超过了对照药叶枯唑, 具有一定 的广谱性. 化合物 $\mathbf{A}_{15}$ 的抗水稻白叶枯病菌的活体测试 结果表明了化合物 $\mathbf{A}_{\mathbf{1 5}}$ 对水稻白叶枯病菌具有良好的体 内治疗和保护作用. 扫描电镜实验表明: 该类化合物对 烟草青枯病菌的抑菌过程可能是通过作用于细菌表面, 导致细菌表面发生皱缩和菌体两端凹陷，从而达到抑菌 的目的; 对水稻白叶枯病菌的抑菌过程可能是通过导致 细菌干癔和卷曲, 导致细菌结构不完整从而达到抑菌的 效果, 进一步验证了化合物的抑菌作用.

\section{3 实验部分}

\section{1 仪器与试剂}

目标化合物的 ${ }^{1} \mathrm{H}$ NMR、 ${ }^{13} \mathrm{C}$ NMR、 ${ }^{19} \mathrm{~F}$ NMR 由 Bruker Ascend-400 型核磁共振波谱仪 (400 MHz) 测得, 以四甲基硅烷(TMS)为内标, $\mathrm{CDCl}_{3}$ 为溶剂; Thermo Scientic Q Exactive 高分辨质谱仪(HRMS, 赛默飞世尔 科技有限公司); XT-4 型数字显微熔点测定仪(北京科德 仪器有限公司); Multiskan FC 酶标仪(赛默飞世尔科技 有限公司); WFH-203B 型三用紫外分析仪(上海精科实 业有限公司); LDZX-50KBS 立式高压蒸汽灭菌器(上海 申安医疗器械厂).

杨梅苷试剂购自重庆铂锶钛科技有限公司, 取代的 邻氨基苯甲酸、1,3-二溴丙烷和 1,4-二溴丁烷等试剂购 自上海泰坦科技股份有限公司; $N, N$-二甲基甲酰胺 $(\mathrm{DMF})$ 和无水乙醇等溶剂均为市售分析纯.

\section{2 实验方法}

\subsection{1 目标化合物 $\mathbf{A}_{1} \sim \mathbf{A}_{22}$ 的合成}

中间体 1 和 2 的合成参照文献[25-26]，中间体 $\mathbf{3} \sim 5$ 的合成参照文献[21, 27].

在 $100 \mathrm{~mL}$ 的单口圆底烧瓶中加入中间体 $\mathbf{5}(1.2$ $\mathrm{mmol}) 、 \mathrm{KOH}(1.5 \mathrm{mmol})$ 和 $20 \mathrm{~mL}$ DMF, 常温搅拌 $0.5 \mathrm{~h}$ 后, 缓慢加入中间体 $2(1 \mathrm{mmol})$ 的 DMF 溶液, 升温至 $60{ }^{\circ} \mathrm{C}$ 并继续摚拌 $8 \sim 10 \mathrm{~h}$. 薄层色谱(TLC) 跟踪反应 $[V$ (石油醚) $: V($ 乙酸乙酯 $)=1: 1]$, 当反应结束, 停止反 应, 反应液用 $100 \mathrm{~mL}$ 的水分散, 再用质量分数为 $5 \%$ 的 稀盐酸调节体系 $\mathrm{pH}$ 至 $5 \sim 6$, 静置后抽滤得粗产物, 经 柱层析 $[V$ (石油醚) $: V$ (乙酸乙酯 $)=3: 1 \sim 1: 2$ ]纯化得 到目标化合物 $\mathbf{A}_{\mathbf{1}} \sim \mathbf{A}_{22}$.

5,7-二甲氧基-3-(4-((6-甲基喹唑啉-4-基)硫基)丁氧 基)-2-(3,4,5-三甲氧基苯基)-4 $\mathrm{H}$-色烯-4-酮 $\left(\mathbf{A}_{1}\right)$ ：黄色固 体, 收率 74.7\%. m.p. 147.8 149.1 ${ }^{\circ} \mathrm{C}$; ${ }^{1} \mathrm{H}$ NMR (400 $\left.\mathrm{MHz}, \mathrm{CDCl}_{3}\right) \delta: 8.89(\mathrm{~s}, 1 \mathrm{H}), 7.83(\mathrm{~d}, J=8.7 \mathrm{~Hz}, 2 \mathrm{H})$, 7.66 (d, $J=8.3 \mathrm{~Hz}, 1 \mathrm{H}), 7.36$ (s, 2H), 6.50 (d, $J=2.2 \mathrm{~Hz}$, $1 \mathrm{H}), 6.35$ (d, $J=2.2 \mathrm{~Hz}, 1 \mathrm{H}), 4.10$ (t, $J=5.7 \mathrm{~Hz}, 2 \mathrm{H}), 3.97$ (s, 3H), $3.95 \sim 3.88(\mathrm{~m}, 12 \mathrm{H}), 3.41(\mathrm{t}, J=6.7 \mathrm{~Hz}, 2 \mathrm{H}), 2.55$ (s, 3H), $1.99 \sim 1.91(\mathrm{~m}, 4 \mathrm{H}) ;{ }^{13} \mathrm{C}$ NMR $\left(101 \mathrm{MHz}, \mathrm{CDCl}_{3}\right)$ $\delta: 174.02,170.52,163.98,161.04,158.79,152.93,152.61$, $146.25,140.63,139.90,137.54,135.73,128.39,126.09$, 123.91, 122.78, 109.43, 105.89, 95.81, 92.42, 71.77, 60.99, $56.37,55.83,29.50,29.15,25.72,21.80$; HRMS calcd for $\mathrm{C}_{33} \mathrm{H}_{35} \mathrm{O}_{8} \mathrm{~N}_{2} \mathrm{~S}[\mathrm{M}+\mathrm{H}]^{+}$619.2109, found 619.2104.

5,7-二甲氧基-3-(4-((8-甲基喹唑啉-4-基)硫基)丁氧 基)-2-(3,4,5-三甲氧基苯基)-4 $\mathrm{H}$-色烯-4-酮 $\left(\mathbf{A}_{2}\right.$ ): 黄色固 体, 收率 75.1\%. m.p. 131.4 132.3 ${ }^{\circ} \mathrm{C} ;{ }^{1} \mathrm{H}$ NMR (400 $\left.\mathrm{MHz}, \mathrm{CDCl}_{3}\right) \delta: 8.95(\mathrm{~s}, 1 \mathrm{H}), 7.87(\mathrm{~d}, J=8.2 \mathrm{~Hz}, 1 \mathrm{H})$, $7.64(\mathrm{~d}, J=7.1 \mathrm{~Hz}, 1 \mathrm{H}), 7.45 \sim 7.38(\mathrm{~m}, 1 \mathrm{H}), 7.28(\mathrm{~s}, 2 \mathrm{H})$, $6.42(\mathrm{~d}, J=2.2 \mathrm{~Hz}, 1 \mathrm{H}), 6.28(\mathrm{~d}, J=2.2 \mathrm{~Hz}, 1 \mathrm{H}), 4.02(\mathrm{t}$, $J=5.8 \mathrm{~Hz}, 2 \mathrm{H}), 3.89(\mathrm{~s}, 3 \mathrm{H}), 3.84(\mathrm{~s}, 6 \mathrm{H}), 3.83(\mathrm{~s}, 6 \mathrm{H})$, $3.36(\mathrm{t}, J=6.8 \mathrm{~Hz}, 2 \mathrm{H}), 2.71(\mathrm{~s}, 3 \mathrm{H}), 1.88(\mathrm{~s}, 4 \mathrm{H}) ;{ }^{13} \mathrm{C}$ NMR $\left(101 \mathrm{MHz}, \mathrm{CDCl}_{3}\right) \delta: 174.00,164.00,161.04$, $158.80,152.97,152.63,151.91,140.59,139.93,136.16$, $134.51,127.35,126.07,123.72,121.70,109.43,105.90$, $95.83,92.45,71.65,61.00,56.39,55.83,29.54,25.62$, 17.91; HRMS calcd for $\mathrm{C}_{33} \mathrm{H}_{35} \mathrm{O}_{8} \mathrm{~N}_{2} \mathrm{~S}[\mathrm{M}+\mathrm{H}]^{+} 619.2109$, found 619.2092 .

5,7-二甲氧基-3-(4-((6-氯喹唑啉-4-基)硫基)丁氧 基)-2-(3,4,5-三甲氧基苯基)-4H-色烯-4-酮( $\mathbf{A}_{\mathbf{3}}$ ): 黄色固 体, 收率 68.7\%. m.p. 118.3 119.2 ${ }^{\circ} \mathrm{C} ;{ }^{1} \mathrm{H}$ NMR (400 $\left.\mathrm{MHz}, \mathrm{CDCl}_{3}\right) \delta: 8.88(\mathrm{~s}, 1 \mathrm{H}), 7.98(\mathrm{dd}, J=12.2,5.5 \mathrm{~Hz}$, $2 \mathrm{H}), 7.74$ (dd, $J=9.0,2.2 \mathrm{~Hz}, 1 \mathrm{H}), 7.27$ (s, 2H), 6.43 (d, $J=2.2 \mathrm{~Hz}, 1 \mathrm{H}), 6.29$ (d, $J=2.2 \mathrm{~Hz}, 1 \mathrm{H}), 4.02$ (t, $J=5.9$ $\mathrm{Hz}, 2 \mathrm{H}), 3.90$ (s, 3H), 3.85 (s, 6H), $3.84(\mathrm{~d}, J=1.1 \mathrm{~Hz}$, $6 \mathrm{H}), 3.39(\mathrm{t}, J=6.9 \mathrm{~Hz}, 2 \mathrm{H}), 1.88(\mathrm{dt}, J=13.0,8.5 \mathrm{~Hz}$, $4 \mathrm{H}) ;{ }^{13} \mathrm{C}$ NMR $\left(101 \mathrm{MHz}, \mathrm{CDCl}_{3}\right) \delta: 174.02,164.04$, $161.03,158.81,152.98,152.57,140.55,139.96,135.24$, $133.67,129.22,126.03,124.33,123.11,109.40,105.92$, $95.85,92.46,71.55,61.00,56.39,55.84,29.77,29.38$, 25.53; HRMS calcd for $\mathrm{C}_{32} \mathrm{H}_{32} \mathrm{O}_{8} \mathrm{~N}_{2} \mathrm{ClS}[\mathrm{M}+\mathrm{H}]^{+}$ 639.1562 , found 639.1546 .

5,7-二甲氧基-3-(4-((6-溴喹唑啉-4-基)硫基)丁氧 基)-2-(3,4,5-三甲氧基苯基)-4 $\mathrm{H}$-色烯-4-酮 $\left(\mathbf{A}_{4}\right.$ ): 黄色固 体, 收率 35.2\%. m.p. 111.4 112.9 ${ }^{\circ} \mathrm{C} ;{ }^{1} \mathrm{H}$ NMR (400 $\left.\mathrm{MHz}, \mathrm{CDCl}_{3}\right) \delta: 8.90(\mathrm{~s}, 1 \mathrm{H}), 8.19(\mathrm{~d}, J=1.9 \mathrm{~Hz}, 1 \mathrm{H})$, $7.98 \sim 7.85(\mathrm{~m}, 2 \mathrm{H}), 7.27(\mathrm{~s}, 2 \mathrm{H}), 6.43(\mathrm{~d}, J=2.2 \mathrm{~Hz}, 1 \mathrm{H})$, $6.29(\mathrm{~d}, J=2.1 \mathrm{~Hz}, 1 \mathrm{H}), 4.02(\mathrm{t}, J=5.8 \mathrm{~Hz}, 2 \mathrm{H}), 3.90(\mathrm{~s}$, $3 \mathrm{H}), 3.86(\mathrm{~s}, 6 \mathrm{H}), 3.84(\mathrm{~s}, 6 \mathrm{H}), 3.41(\mathrm{t}, J=6.9 \mathrm{~Hz}, 2 \mathrm{H})$, 1.88 (ddd, $J=17.3,12.0,5.4 \mathrm{~Hz}, 4 \mathrm{H}$ ); ${ }^{13} \mathrm{C}$ NMR $(101$ $\left.\mathrm{MHz}, \mathrm{CDCl}_{3}\right) \delta: 174.01,164.05,161.04,158.82,152.99$, $152.74,152.01,140.54,139.97,138.13,128.78,126.47$, $126.02,124.68,121.95,109.41,105.93,95.87,92.47$, 
71.51, 61.02, 56.41, 55.85, 29.92, 29.35, 25.50; HRMS calcd for $\mathrm{C}_{32} \mathrm{H}_{32} \mathrm{O}_{8} \mathrm{~N}_{2} \mathrm{BrS}[\mathrm{M}+\mathrm{H}]^{+}$683.1057, found 683.1059 .

5,7-二甲氧基-3-(4-((6-氟喹唑啉-4-基)硫基)丁氧 基)-2-(3,4,5-三甲氧基苯基)- $4 H$-色烯-4-酮( $\mathbf{A}_{5}$ ): 黄色固 体, 收率 65.3\%. m.p. 106.2 108.1 ${ }^{\circ} \mathrm{C} ;{ }^{1} \mathrm{H}$ NMR (400 $\left.\mathrm{MHz}, \mathrm{CDCl}_{3}\right) \delta: 8.92(\mathrm{~s}, 1 \mathrm{H}), 7.96(\mathrm{dd}, J=9.2,5.2 \mathrm{~Hz}$, $1 \mathrm{H}), 7.68(\mathrm{dd}, J=8.8,2.7 \mathrm{~Hz}, 1 \mathrm{H}), 7.63 \sim 7.57(\mathrm{~m}, 1 \mathrm{H})$, $7.35(\mathrm{~s}, 2 \mathrm{H}), 6.50(\mathrm{~d}, J=2.2 \mathrm{~Hz}, 1 \mathrm{H}), 6.36(\mathrm{~d}, J=2.2 \mathrm{~Hz}$, $1 \mathrm{H}), 4.09$ (t, $J=5.8 \mathrm{~Hz}, 2 \mathrm{H}), 3.97$ (s, 3H), 3.93 (s, 6H), $3.92(\mathrm{~d}, J=1.9 \mathrm{~Hz}, 6 \mathrm{H}), 3.42$ (t, $J=6.8 \mathrm{~Hz}, 2 \mathrm{H}), 2.00 \sim$ $1.89(\mathrm{~m}, 4 \mathrm{H}) ;{ }^{13} \mathrm{C}$ NMR (101 $\left.\mathrm{MHz}, \mathrm{CDCl}_{3}\right) \delta: 174.02$, $170.93\left(\mathrm{~d}, J_{\mathrm{C}-\mathrm{F}}=5.6 \mathrm{~Hz}\right), 164.00,161.45,161.03,158.88$ $\left(\mathrm{d}, J_{\mathrm{C}-\mathrm{F}}=16.3 \mathrm{~Hz}\right), 153.18 \sim 152.87,152.67,144.91$, $140.58,139.91,131.46\left(\mathrm{~d}, J_{\mathrm{C}-\mathrm{F}}=8.7 \mathrm{~Hz}\right), 126.06,124.50$ $\left(\mathrm{d}, J_{\mathrm{C}-\mathrm{F}}=9.0 \mathrm{~Hz}\right), 123.77,123.51,109.42,107.97,107.74$, 105.89, 95.82, 92.41, 71.67, 61.00, 56.37, 55.82, 29.42, $25.61 ;{ }^{19} \mathrm{~F}$ NMR $\left(376 \mathrm{MHz}, \mathrm{CDCl}_{3}\right) \delta:-109.55(\mathrm{td}, J=$ 8.5, $5.3 \mathrm{~Hz}$ ); HRMS calcd for $\mathrm{C}_{32} \mathrm{H}_{32} \mathrm{O}_{8} \mathrm{~N}_{2} \mathrm{FS}[\mathrm{M}+\mathrm{H}]^{+}$ 623.1858 , found 623.1846 .

5,7-二甲氧基-3-(4-((7-氯喹唑啉-4-基)硫基)丁氧 基)-2-(3,4,5-三甲氧基苯基)- $4 H$-色烯-4-酮( $\mathbf{A}_{6}$ ): 黄色固 体, 收率 44.4\%. m.p. 133.1 135.0 ${ }^{\circ} \mathrm{C} ;{ }^{1} \mathrm{H}$ NMR (400 $\left.\mathrm{MHz}, \mathrm{CDCl}_{3}\right) \delta: 8.86(\mathrm{~s}, 1 \mathrm{H}), 8.01 \sim 7.92(\mathrm{~m}, 2 \mathrm{H}), 7.48$ $(\mathrm{dd}, J=8.9,1.9 \mathrm{~Hz}, 1 \mathrm{H}), 7.27(\mathrm{~s}, 2 \mathrm{H}), 6.43(\mathrm{~d}, J=2.2 \mathrm{~Hz}$, $1 \mathrm{H}), 6.28$ (d, $J=2.1 \mathrm{~Hz}, 1 \mathrm{H}), 4.01(\mathrm{t}, J=5.8 \mathrm{~Hz}, 2 \mathrm{H}), 3.89$ (s, 3H), $3.84(\mathrm{~d}, J=3.6 \mathrm{~Hz}, 12 \mathrm{H}), 3.38(\mathrm{t}, J=6.9 \mathrm{~Hz}, 2 \mathrm{H})$, $1.88(\mathrm{dt}, J=13.0,7.0 \mathrm{~Hz}, 4 \mathrm{H}) ;{ }^{13} \mathrm{C}$ NMR $(101 \mathrm{MHz}$, $\left.\mathrm{CDCl}_{3}\right) \quad \delta: 174.00,164.03,161.01,158.79$, 153.07, $152.96 \sim 152.86,152.68,140.62,139.91,128.90,126.62$, $126.03,125.43,122.13,109.38,105.87,95.85,92.45$, 71.54, 61.01, 56.39, 55.86, 29.67, 29.41, 25.55; HRMS calcd for $\mathrm{C}_{32} \mathrm{H}_{32} \mathrm{O}_{8} \mathrm{~N}_{2} \mathrm{ClS}[\mathrm{M}+\mathrm{H}]^{+}$639.1562, found 639.1545 .

5,7-二甲氧基-3-(4-((6-碘喹唑啉-4-基)硫基)丁氧 基)-2-(3,4,5-三甲氧基苯基)- $4 H$-色烯-4-酮 $\left(\mathbf{A}_{7}\right)$ : 黄色固 体, 收率 75.2\%. m.p. 141.2 141.9 ${ }^{\circ} \mathrm{C} ;{ }^{1} \mathrm{H}$ NMR (400 $\left.\mathrm{MHz}, \mathrm{CDCl}_{3}\right) \delta: 8.89(\mathrm{~s}, 1 \mathrm{H}), 8.37(\mathrm{~s}, 1 \mathrm{H}), 8.03(\mathrm{~d}, J=8.8$ $\mathrm{Hz}, 1 \mathrm{H}), 7.71$ (d, $J=8.8 \mathrm{~Hz}, 1 \mathrm{H}), 7.27$ (s, 2H), 6.43 (s, $1 \mathrm{H}), 6.29$ (s, 1H), 4.01 (t, $J=4.9 \mathrm{~Hz}, 2 \mathrm{H}), 3.90$ (d, $J=2.1$ $\mathrm{Hz}, 3 \mathrm{H}), 3.85$ (dd, $J=5.9,1.7 \mathrm{~Hz}, 12 \mathrm{H}), 3.38$ (t, $J=5.9$ $\mathrm{Hz}, 2 \mathrm{H}), 1.87(\mathrm{~s}, 4 \mathrm{H}) ;{ }^{13} \mathrm{C}$ NMR (101 MHz, $\left.\mathrm{CDCl}_{3}\right) \delta$ : $174.02,164.03,161.03,158.80,152.98,152.72,143.04$, $140.56,139.94,132.93,129.26,126.03,125.26,109.40$, $105.90,95.86,92.98,92.45,71.58,61.03,56.41,55.86$,
29.71, 29.38, 25.55; HRMS calcd for $\mathrm{C}_{32} \mathrm{H}_{32} \mathrm{O}_{8} \mathrm{~N}_{2} \mathrm{IS}$ [M+ $\mathrm{H}]^{+}$731.0919, found 731.0902 .

5,7-二甲氧基-3-(4-((6-氯-8-甲基喹唑啉-4-基)硫基) 丁氧基)-2-(3,4,5-三甲氧基苯基)- $4 H$-色烯-4-酮( $\mathbf{A}_{8}$ ): 黄 色固体, 收率 53.0\%. m.p. 69.8 71.0 ${ }^{\circ} \mathrm{C} ;{ }^{1} \mathrm{H}$ NMR (400 $\left.\mathrm{MHz}, \mathrm{CDCl}_{3}\right) \delta: 8.92(\mathrm{~s}, 1 \mathrm{H}), 7.84(\mathrm{~d}, J=1.9 \mathrm{~Hz}, 1 \mathrm{H})$, $7.58(\mathrm{~s}, 1 \mathrm{H}), 7.27$ (s, 2H), 6.43 (d, $J=2.2 \mathrm{~Hz}, 1 \mathrm{H}), 6.28$ (d, $J=2.1 \mathrm{~Hz}, 1 \mathrm{H}), 4.01(\mathrm{t}, J=5.7 \mathrm{~Hz}, 2 \mathrm{H}), 3.89(\mathrm{~s}, 3 \mathrm{H}), 3.85$ $(\mathrm{d}, J=4.6 \mathrm{~Hz}, 12 \mathrm{H}), 3.37(\mathrm{t}, J=6.7 \mathrm{~Hz}, 2 \mathrm{H}), 2.68(\mathrm{~s}, 3 \mathrm{H})$, $1.95 \sim 1.81(\mathrm{~m}, 4 \mathrm{H}) ;{ }^{13} \mathrm{C} \mathrm{NMR}\left(101 \mathrm{MHz}, \mathrm{CDCl}_{3}\right) \delta$ : $174.00,164.01,161.03,158.80,152.97,152.67,152.06$, $140.56,139.92,138.69,134.94,132.96,126.05,124.32$, 120.68, 109.41, 105.88, 95.83, 92.44, 71.60, 61.01, 56.39, 55.84, 29.79, 29.40, 25.56, 17.78; HRMS calcd for $\mathrm{C}_{33} \mathrm{H}_{34} \mathrm{O}_{8} \mathrm{~N}_{2} \mathrm{ClS}[\mathrm{M}+\mathrm{H}]^{+}$653.1719, found 653.1702.

5,7-二甲氧基-3-(4-((7-氟喹唑啉-4-基)硫基)丁氧 基)-2-(3,4,5-三甲氧基苯基)-4H-色烯-4-酮( $\mathbf{A}_{9}$ )：黄色固 体, 收率 34.3\%. m.p. 119.5 120.3 ${ }^{\circ} \mathrm{C} ;{ }^{1} \mathrm{H}$ NMR (400 $\left.\mathrm{MHz}, \mathrm{CDCl}_{3}\right) \delta: 8.83(\mathrm{~s}, 1 \mathrm{H}), 8.01(\mathrm{dd}, J=9.2,5.8 \mathrm{~Hz}$, $1 \mathrm{H}), 7.47$ (dd, $J=9.6,2.5 \mathrm{~Hz}, 1 \mathrm{H}), 7.28$ (s, 2H), 7.24 (dd, $J=8.3,2.6 \mathrm{~Hz}, 1 \mathrm{H}), 6.42(\mathrm{~d}, J=2.2 \mathrm{~Hz}, 1 \mathrm{H}), 6.28(\mathrm{~d}, J=$ $2.2 \mathrm{~Hz}, 1 \mathrm{H}), 4.01$ (t, $J=5.8 \mathrm{~Hz}, 2 \mathrm{H}), 3.89$ (s, 3H), 3.85 (s, $6 \mathrm{H}), 3.84(\mathrm{~s}, 3 \mathrm{H}), 3.84(\mathrm{~s}, 3 \mathrm{H}), 3.34(\mathrm{t}, J=6.8 \mathrm{~Hz}, 2 \mathrm{H})$, $1.93 \sim 1.83(\mathrm{~m}, 4 \mathrm{H}) ;{ }^{13} \mathrm{C} \mathrm{NMR}\left(101 \mathrm{MHz}, \mathrm{CDCl}_{3}\right) \delta$ : $174.01,171.43,166.70,164.08\left(\mathrm{~d}, J_{\mathrm{C}-\mathrm{F}}=16.4 \mathrm{~Hz}\right), 161.03$, $158.79,154.38,152.97,152.63,149.82\left(\mathrm{~d}, J_{\mathrm{C}-\mathrm{F}}=13.4 \mathrm{~Hz}\right)$, $140.60,139.91,126.65\left(\mathrm{~d}, J_{\mathrm{C}-\mathrm{F}}=10.4 \mathrm{~Hz}\right), 126.07,121.12$, $117.57,117.32,112.82,112.61,109.41,105.88,95.82$, $92.42,71.68,61.01,56.38,55.83,29.39,25.67 ;{ }^{19} \mathrm{~F}$ NMR $\left(376 \mathrm{MHz}, \mathrm{CDCl}_{3}\right) \delta$ : $-102.79(\mathrm{td}, J=8.7,5.9 \mathrm{~Hz})$; HRMS calcd for $\mathrm{C}_{32} \mathrm{H}_{32} \mathrm{O}_{8} \mathrm{~N}_{2} \mathrm{FS}[\mathrm{M}+\mathrm{H}]^{+}$623.1858, found 623.1846 .

5,7-二甲氧基-3-(4-((6,7-二甲氧基喹唑啉-4-基)硫 基)丁氧基)-2-(3,4,5-三甲氧基苯基)- $4 H$-色烯-4-酮( $\left.\mathbf{A}_{10}\right)$ : 黄色固体，收率 $28.6 \%$. m.p. 166.2 168.1 ${ }^{\circ} \mathrm{C} ;{ }^{1} \mathrm{H}$ NMR $\left(400 \mathrm{MHz}, \mathrm{CDCl}_{3}\right) \delta: 8.74(\mathrm{~s}, 1 \mathrm{H}), 7.28(\mathrm{~s}, 2 \mathrm{H}), 7.17$ (s, $1 \mathrm{H}), 7.13$ (s, 1H), 6.42 (d, $J=2.3 \mathrm{~Hz}, 1 \mathrm{H}), 6.28$ (d, $J=2.2$ $\mathrm{Hz}, 1 \mathrm{H}), 4.02$ (t, $J=5.7 \mathrm{~Hz}, 2 \mathrm{H}), 3.97(\mathrm{~d}, J=2.2 \mathrm{~Hz}, 6 \mathrm{H})$, 3.89 (s, 3H), $3.84(\mathrm{~d}, J=2.3 \mathrm{~Hz}, 12 \mathrm{H}), 3.33(\mathrm{t}, J=5.8 \mathrm{~Hz}$, $2 \mathrm{H}), 1.88(\mathrm{~s}, 4 \mathrm{H}) ;{ }^{13} \mathrm{C} \mathrm{NMR}\left(101 \mathrm{MHz}, \mathrm{CDCl}_{3}\right) \delta: 174.02$, $167.56,163.98,161.04,158.79,155.42,152.97,152.54$, 149.89 , 145.48, 140.65, 139.90, 126.10, 119.17, 109.43, 107.10, 105.88, 101.60, 95.81, 92.42, 71.79, 61.00, 56.35, 55.83, 29.53, 29.23, 25.85; HRMS calcd for $\mathrm{C}_{34} \mathrm{H}_{37} \mathrm{O}_{10} \mathrm{~N}_{2} \mathrm{~S}$ $[\mathrm{M}+\mathrm{H}]^{+} \quad 665.2163$, found 665.2155 . 
5,7-二甲氧基-3-(4-((7,8-二甲基喹唑啉-4-基)硫基) 丁氧基)-2-(3,4,5-三甲氧基苯基)- $4 H$-色烯-4-酮 $\left(\mathbf{A}_{11}\right)$ : 黄 色固体, 收率 31.3\%. m.p. 179.9 181.3 ${ }^{\circ} \mathrm{C} ;{ }^{1} \mathrm{H}$ NMR $(400$ $\left.\mathrm{MHz}, \mathrm{CDCl}_{3}\right) \delta: 8.87(\mathrm{~s}, 1 \mathrm{H}), 7.74(\mathrm{~d}, J=8.5 \mathrm{~Hz}, 1 \mathrm{H})$, $7.31 \sim 7.24(\mathrm{~m}, 3 \mathrm{H}), 6.42(\mathrm{~d}, J=2.2 \mathrm{~Hz}, 1 \mathrm{H}), 6.27$ (d, $J=$ $2.2 \mathrm{~Hz}, 1 \mathrm{H}), 4.02(\mathrm{t}, J=5.7 \mathrm{~Hz}, 2 \mathrm{H}), 3.89(\mathrm{~s}, 3 \mathrm{H}), 3.83(\mathrm{t}$, $J=2.6 \mathrm{~Hz}, 12 \mathrm{H}), 3.31(\mathrm{t}, J=6.6 \mathrm{~Hz}, 2 \mathrm{H}), 2.59(\mathrm{~s}, 3 \mathrm{H})$, 2.42 (s, 3H), 1.87 (t, $J=2.4 \mathrm{~Hz}, 4 \mathrm{H}) ;{ }^{13} \mathrm{C}$ NMR $(101 \mathrm{MHz}$, $\left.\mathrm{CDCl}_{3}\right) \delta: 174.01,171.09,163.97,161.04,158.79,152.96$, $152.57,146.86,141.95,140.64,139.88,134.31,129.65$, $126.11,122.26,120.69,109.44,105.86,95.80,92.41$, $71.80,61.01,56.37,55.83,29.54,29.20,25.75,20.96$, 13.08; HRMS calcd for $\mathrm{C}_{34} \mathrm{H}_{37} \mathrm{O}_{8} \mathrm{~N}_{2} \mathrm{~S}[\mathrm{M}+\mathrm{H}]^{+}$633.2265, found 633.2257 .

5,7-二甲氧基-3-(3-((6-甲基喹唑啉-4-基)硫基)丙氧 基)-2-(3,4,5-三甲氧基苯基)-4H-色烯-4-酮( $\mathbf{A}_{\mathbf{1 2}}$ ): 白色固 体, 收率 39.9\%. m.p. 78.4 80.1 ${ }^{\circ} \mathrm{C} ;{ }^{1} \mathrm{H}$ NMR $(400 \mathrm{MHz}$, $\left.\mathrm{CDCl}_{3}\right) \delta: 8.87(\mathrm{~s}, 1 \mathrm{H}), 7.82(\mathrm{t}, J=5.3 \mathrm{~Hz}, 2 \mathrm{H}), 7.66(\mathrm{dd}$, $J=8.6,1.8 \mathrm{~Hz}, 1 \mathrm{H}), 7.36(\mathrm{~s}, 2 \mathrm{H}), 6.51(\mathrm{~d}, J=2.2 \mathrm{~Hz}, 1 \mathrm{H})$, $6.37(\mathrm{~d}, J=2.2 \mathrm{~Hz}, 1 \mathrm{H}), 4.20(\mathrm{t}, J=6.0 \mathrm{~Hz}, 2 \mathrm{H}), 3.98(\mathrm{~s}$, $3 \mathrm{H}$ ), 3.93 (s, 6H), 3.92 (d, $J=1.3 \mathrm{~Hz}, 6 \mathrm{H}), 3.52$ (t, $J=7.1$ $\mathrm{Hz}, 2 \mathrm{H}), 2.54$ (s, 3H), 2.28 2.19 (m, 2H); ${ }^{13} \mathrm{C}$ NMR (101 $\left.\mathrm{MHz}, \mathrm{CDCl}_{3}\right) \delta: 173.97,170.34,164.01,161.03,158.81$, $153.12 \sim 152.70,146.24,140.47,139.94,137.53,135.73$, $128.37,126.02,123.90,122.79,109.41,105.89,95.84$, $92.42,70.95,60.99,56.37,55.83,29.97,26.24,21.77$; HRMS calcd for $\mathrm{C}_{32} \mathrm{H}_{33} \mathrm{O}_{8} \mathrm{~N}_{2} \mathrm{~S}[\mathrm{M}+\mathrm{H}]^{+}$605.1952, found 605.1945.

5,7-二甲氧基-3-(3-((8-甲基喹唑啉-4-基)硫基)丙氧 基)-2-(3,4,5-三甲氧基苯基)-4H-色烯-4-酮 $\left(\mathbf{A}_{13}\right.$ ): 黄色固 体, 收率 48.4\%. m.p. $138.8 \sim 140.2{ }^{\circ} \mathrm{C} ;{ }^{1} \mathrm{H}$ NMR (400 $\left.\mathrm{MHz}, \mathrm{CDCl}_{3}\right) \delta: 8.96(\mathrm{~s}, 1 \mathrm{H}), 7.87(\mathrm{~d}, J=8.3 \mathrm{~Hz}, 1 \mathrm{H})$, $7.65(\mathrm{~d}, J=7.1 \mathrm{~Hz}, 1 \mathrm{H}), 7.45 \sim 7.39(\mathrm{~m}, 1 \mathrm{H}), 7.27(\mathrm{~s}, 2 \mathrm{H})$, $6.44(\mathrm{~d}, J=2.2 \mathrm{~Hz}, 1 \mathrm{H}), 6.29$ (d, $J=2.2 \mathrm{~Hz}, 1 \mathrm{H}), 4.12$ (t, $J=5.9 \mathrm{~Hz}, 2 \mathrm{H}), 3.90(\mathrm{~s}, 3 \mathrm{H}), 3.86(\mathrm{~s}, 6 \mathrm{H}), 3.84(\mathrm{~s}, 3 \mathrm{H})$, $3.84(\mathrm{~s}, 3 \mathrm{H}), 3.50$ (t, $J=7.1 \mathrm{~Hz}, 2 \mathrm{H}), 2.72(\mathrm{~s}, 3 \mathrm{H}), 2.20 \sim$ $2.12(\mathrm{~m}, 2 \mathrm{H}) ;{ }^{13} \mathrm{C}$ NMR $\left(101 \mathrm{MHz}, \mathrm{CDCl}_{3}\right) \delta:$ 173.94, $164.06,161.05,158.83,152.95,151.71,140.42,140.00$, $135.93,134.76,127.51,125.99,123.67,121.76,109.41$, 105.92, 95.88, 92.47, 70.79, 61.01, 56.41, 55.85, 29.86, 26.87, 17.97; HRMS calcd for $\mathrm{C}_{32} \mathrm{H}_{33} \mathrm{O}_{8} \mathrm{~N}_{2} \mathrm{~S}[\mathrm{M}+\mathrm{H}]^{+}$ 605.1952, found 605.1937.

5,7-二甲氧基-3-(3-((6-氯喹唑啉-4-基)硫基)丙氧 基)-2-(3,4,5-三甲氧基苯基)-4H-色烯-4-酮 $\left(\mathbf{A}_{14}\right)$ ：黄色固 体, 收率 57.7\%. m.p. 178.2 180.0 ${ }^{\circ} \mathrm{C} ;{ }^{1} \mathrm{H}$ NMR (400
$\left.\mathrm{MHz}, \mathrm{CDCl}_{3}\right) \delta: 8.88(\mathrm{~s}, 1 \mathrm{H}), 8.01(\mathrm{t}, J=6.2 \mathrm{~Hz}, 2 \mathrm{H}), 7.75$ (dd, $J=8.9,2.2 \mathrm{~Hz}, 1 \mathrm{H}), 7.26$ (s, 2H), 6.44 (d, $J=2.2 \mathrm{~Hz}$, $1 \mathrm{H}), 6.30$ (d, $J=2.2 \mathrm{~Hz}, 1 \mathrm{H}), 4.11(\mathrm{t}, J=5.8 \mathrm{~Hz}, 2 \mathrm{H}), 3.91$ (s, 3H), 3.86 (s, 6H), $3.84(\mathrm{~d}, J=2.6 \mathrm{~Hz}, 6 \mathrm{H}), 3.55$ (t, $J=$ $7.0 \mathrm{~Hz}, 2 \mathrm{H}), 2.20 \sim 2.09(\mathrm{~m}, 2 \mathrm{H}) ;{ }^{13} \mathrm{C}$ NMR $(101 \mathrm{MHz}$, $\left.\mathrm{CDCl}_{3}\right) \delta: 173.94,164.11,161.04,158.85,153.03,151.91$, $140.32,140.04,135.54,133.97,128.73,125.93,124.25$, $123.18,109.39,105.94,95.91,92.49,70.53,61.01,56.42$, 55.86, 29.65, 27.18; HRMS calcd for $\mathrm{C}_{31} \mathrm{H}_{30} \mathrm{O}_{8} \mathrm{~N}_{2} \mathrm{ClS}$ $[\mathrm{M}+\mathrm{H}]^{+} 625.1406$, found 625.1390 .

5,7-二甲氧基-3-(3-((6-澳喹唑啉-4-基)硫基)丙氧 基)-2-(3,4,5-三甲氧基苯基)-4 $\mathrm{H}$-色烯-4-酮( $\mathbf{A}_{\mathbf{1 5}}$ )：黄色固 体, 收率 47.6\%. m.p. 199.6 200.8 ${ }^{\circ} \mathrm{C} ;{ }^{1} \mathrm{H}$ NMR (400 $\left.\mathrm{MHz}, \mathrm{CDCl}_{3}\right) \delta: 8.92(\mathrm{~s}, 1 \mathrm{H}), 8.20(\mathrm{~d}, J=2.0 \mathrm{~Hz}, 1 \mathrm{H})$, 7.89 (dd, $J=8.9,2.1 \mathrm{~Hz}, 1 \mathrm{H}), 7.80$ (d, $J=8.9 \mathrm{~Hz}, 1 \mathrm{H})$, 7.35 (s, 2H), 6.51 (d, $J=2.2 \mathrm{~Hz}, 1 \mathrm{H}), 6.37$ (d, $J=2.2 \mathrm{~Hz}$, $1 \mathrm{H}), 4.20$ (t, $J=5.9 \mathrm{~Hz}, 2 \mathrm{H}), 3.99$ (s, 3H), 3.94 (s, 6H), 3.92 (s, 6H), 3.55 (t, $J=7.1 \mathrm{~Hz}, 2 \mathrm{H}), 2.26 \sim 2.19$ (m, 2H); ${ }^{13} \mathrm{C}$ NMR (101 MHz, $\left.\mathrm{CDCl}_{3}\right) \delta: 173.93,170.53,164.02$, 161.02, 158.81, 153.70, 152.92, 146.60, 140.40, 139.95, $137.05,130.53,126.33,125.98,124.97,120.85,109.39$, $105.87,95.85,92.42,70.74,61.01,56.39,55.83,29.81$, 26.57; HRMS calcd for $\mathrm{C}_{31} \mathrm{H}_{30} \mathrm{O}_{8} \mathrm{~N}_{2} \mathrm{BrS}[\mathrm{M}+\mathrm{H}]^{+}$ 669.0901, found 669.0886.

5,7-二甲氧基-3-(3-((6-氟喹唑啉-4-基)硫基)丙氧 基)-2-(3,4,5-三甲氧基苯基)-4H-色烯-4-酮( $\mathbf{A}_{\mathbf{1 6}}$ ): 白色固 体, 收率 50.5\% m.p. 79.0 79.7 ${ }^{\circ} \mathrm{C} ;{ }^{1} \mathrm{H}$ NMR $(400 \mathrm{MHz}$, $\left.\mathrm{CDCl}_{3}\right) \delta: 8.91(\mathrm{~s}, 1 \mathrm{H}), 7.95(\mathrm{dd}, J=9.2,5.2 \mathrm{~Hz}, 1 \mathrm{H}), 7.66$ (dd, $J=8.8,2.7 \mathrm{~Hz}, 1 \mathrm{H}), 7.62 \sim 7.56(\mathrm{~m}, 1 \mathrm{H}), 7.35(\mathrm{~s}, 2 \mathrm{H})$, $6.51(\mathrm{~d}, J=2.2 \mathrm{~Hz}, 1 \mathrm{H}), 6.37(\mathrm{~d}, J=2.2 \mathrm{~Hz}, 1 \mathrm{H}), 4.20(\mathrm{t}$, $J=5.9 \mathrm{~Hz}, 2 \mathrm{H}), 3.98$ (s, 3H), 3.94 (d, $J=4.8 \mathrm{~Hz}, 6 \mathrm{H}), 3.92$ (s, 6H), 3.54 (t, $J=7.1 \mathrm{~Hz}, 2 \mathrm{H}), 2.28 \sim 2.19(\mathrm{~m}, 2 \mathrm{H}) ;{ }^{13} \mathrm{C}$ NMR $\left(101 \mathrm{MHz}, \mathrm{CDCl}_{3}\right) \delta: 173.95,170.79\left(\mathrm{~d}, J_{\mathrm{C}-\mathrm{F}}=5.5\right.$ $\mathrm{Hz}), 164.03,161.44,161.04,158.88\left(\mathrm{~d}, J_{\mathrm{C}-\mathrm{F}}=11.9 \mathrm{~Hz}\right)$, $153.17 \sim 152.75,144.90,140.43,139.98,131.46\left(\mathrm{~d}, J_{\mathrm{C}-\mathrm{F}}=\right.$ $8.7 \mathrm{~Hz}), 125.99,124.49$ (d, $\left.J_{\mathrm{C}-\mathrm{F}}=9.1 \mathrm{~Hz}\right), 123.77,123.52$, 109.41, 107.98, 107.75, 105.91, 95.86, 92.44, 70.82, 61.00, $56.39,55.83,29.84,26.56 ;{ }^{19} \mathrm{~F}$ NMR (376 MHz, $\left.\mathrm{CDCl}_{3}\right) \delta$ : -109.55 (td, $J=8.4,5.3 \mathrm{~Hz}$ ); HRMS calcd for $\mathrm{C}_{31} \mathrm{H}_{30} \mathrm{O}_{8^{-}}$ $\mathrm{N}_{2} \mathrm{FS}[\mathrm{M}+\mathrm{H}]^{+}$609.1701, found 609.1690.

5,7-二甲氧基-3-(3-((7-氯喹唑啉-4-基)硫基)丙氧 基)-2-(3,4,5-三甲氧基苯基)-4 $\mathrm{H}$-色烯-4-酮 $\left(\mathbf{A}_{\mathbf{1 7}}\right.$ ): 白色固 体, 收率 38.1\%. m.p. 166.3 168.5 ${ }^{\circ} \mathrm{C} ;{ }^{1} \mathrm{H}$ NMR $(400$ $\left.\mathrm{MHz}, \mathrm{CDCl}_{3}\right) \delta: 8.85(\mathrm{~s}, 1 \mathrm{H}), 8.00 \sim 7.92(\mathrm{~m}, 2 \mathrm{H}), 7.48(\mathrm{~d}$, $J=8.6 \mathrm{~Hz}, 1 \mathrm{H}), 7.26(\mathrm{~s}, 2 \mathrm{H}), 6.44(\mathrm{~s}, 1 \mathrm{H}), 6.30(\mathrm{~s}, 1 \mathrm{H})$, 
4.11 (s, 2H), 3.91 (s, 3H), 3.86 (s, 6H), 3.84 (d, $J=1.2 \mathrm{~Hz}$, $6 \mathrm{H}), 3.52(\mathrm{t}, J=6.3 \mathrm{~Hz}, 2 \mathrm{H}), 2.15(\mathrm{~s}, 2 \mathrm{H}) ;{ }^{13} \mathrm{C}$ NMR $(101$ $\left.\mathrm{MHz}, \mathrm{CDCl}_{3}\right) \delta: 173.94,164.09,161.04,158.84,153.44 \sim$ $152.81,140.68,140.36,140.01,128.88,126.67,125.96$, $125.47,122.16,109.40,105.92,95.91,92.49,70.68,61.03$, $56.43,55.88,29.81,26.90$; HRMS calcd for $\mathrm{C}_{31} \mathrm{H}_{30} \mathrm{O}_{8^{-}}$ $\mathrm{N}_{2} \mathrm{ClS}[\mathrm{M}+\mathrm{H}]^{+}$625.1406, found 625.1390.

5,7-二甲氧基-3-(3-((6-碘喹唑啉-4-基)硫基)丙氧 基)-2-(3,4,5-三甲氧基苯基)-4 $\mathrm{H}$-色烯-4-酮( $\mathbf{A}_{\mathbf{1 8}}$ ): 黄色固 体, 收率 33.2\%. m.p. 195.1 196.2 ${ }^{\circ} \mathrm{C} ;{ }^{1} \mathrm{H}$ NMR (400 $\left.\mathrm{MHz}, \mathrm{CDCl}_{3}\right) \delta: 8.92(\mathrm{~s}, 1 \mathrm{H}), 8.41(\mathrm{~d}, J=1.8 \mathrm{~Hz}, 1 \mathrm{H})$, $8.06(\mathrm{dd}, J=8.8,1.9 \mathrm{~Hz}, 1 \mathrm{H}), 7.65(\mathrm{~d}, J=8.8 \mathrm{~Hz}, 1 \mathrm{H})$, 7.35 (s, 2H), $6.51(\mathrm{~d}, J=2.2 \mathrm{~Hz}, 1 \mathrm{H}), 6.37(\mathrm{~d}, J=2.2 \mathrm{~Hz}$, 1H), 4.20 (t, $J=5.9 \mathrm{~Hz}, 2 \mathrm{H}), 3.99$ (s, 3H), 3.94 (s, 6H), $3.92(\mathrm{~d}, J=1.2 \mathrm{~Hz}, 6 \mathrm{H}), 3.55(\mathrm{t}, J=7.0 \mathrm{~Hz}, 2 \mathrm{H}), 2.26 \sim$ $2.18(\mathrm{~m}, 2 \mathrm{H}) ;{ }^{13} \mathrm{C}$ NMR $\left(101 \mathrm{MHz}, \mathrm{CDCl}_{3}\right) \delta: 173.92$, $170.22,164.01,161.02,158.80,153.75,152.91,146.94$, $142.36,140.39,139.94,132.84,130.38,125.97,125.46$, 109.39, 105.87, 95.84, 92.35, 70.73, 61.01, 56.39, 55.83, 29.82, 26.55; HRMS calcd for $\mathrm{C}_{31} \mathrm{H}_{30} \mathrm{O}_{8} \mathrm{~N}_{2} \mathrm{IS}[\mathrm{M}+\mathrm{H}]^{+}$, found 717.0741 .

5,7-二甲氧基-3-(3-((6-氯-8-甲基喹唑啉-4-基)硫基) 丙氧基)-2-(3,4,5-三甲氧基苯基)- $4 H$-色烯-4-酮( $\mathbf{A}_{\mathbf{1 9}}$ ): 黄 色固体, 收率 39.4\%. m.p. 156.0 157.2 ${ }^{\circ} \mathrm{C} ;{ }^{1} \mathrm{H}$ NMR $\left(400 \mathrm{MHz}, \mathrm{CDCl}_{3}\right) \delta: 8.88(\mathrm{~s}, 1 \mathrm{H}), 7.80(\mathrm{~s}, 1 \mathrm{H}), 7.55(\mathrm{~s}$, $1 \mathrm{H}), 7.27$ (s, 2H), 6.43 (s, 1H), 6.29 (s, 1H), $4.12(\mathrm{~s}, 2 \mathrm{H})$, 3.91 (s, 3H), $3.86(\mathrm{~s}, 6 \mathrm{H}), 3.84(\mathrm{~s}, 6 \mathrm{H}), 3.47$ (s, 2H), 2.65 (s, 3H), 2.15 (s, 2H); ${ }^{13} \mathrm{C}$ NMR (101 MHz, $\left.\mathrm{CDCl}_{3}\right) \delta$ : 173.96, 164.06, 161.05, 158.84, 152.97, 152.53, 140.43, $140.02,139.17,134.50,132.59,125.99,124.44,120.64$, 109.43, 105.95, 95.89, 92.48, 70.82, 61.03, 56.46, 55.88, 29.85, 26.80, 17.70; HRMS calcd for $\mathrm{C}_{32} \mathrm{H}_{32} \mathrm{O}_{8} \mathrm{~N}_{2} \mathrm{ClS}$ $[\mathrm{M}+\mathrm{H}]^{+}$639.1562, found 639.1550.

5,7-二甲氧基-3-(3-((7-氟喹唑啉-4-基)硫基)丙氧 基)-2-(3,4,5-三甲氧基苯基)-4H-色烯-4-酮 $\left(\mathbf{A}_{\mathbf{2 0}}\right.$ ): 黄色固 体, 收率 $40.2 \%$. m.p. $147.9 \sim 149.2{ }^{\circ} \mathrm{C} ;{ }^{1} \mathrm{H}$ NMR (400 $\left.\mathrm{MHz}, \mathrm{CDCl}_{3}\right) \delta: 8.82(\mathrm{~s}, 1 \mathrm{H}), 8.01(\mathrm{dd}, J=9.2,5.8 \mathrm{~Hz}$, $1 \mathrm{H}), 7.47(\mathrm{dd}, J=9.6,2.5 \mathrm{~Hz}, 1 \mathrm{H}), 7.30 \sim 7.22(\mathrm{~m}, 3 \mathrm{H})$, $6.44(\mathrm{~d}, J=2.2 \mathrm{~Hz}, 1 \mathrm{H}), 6.30(\mathrm{~d}, J=2.2 \mathrm{~Hz}, 1 \mathrm{H}), 4.12(\mathrm{t}$, $J=6.0 \mathrm{~Hz}, 2 \mathrm{H}), 3.91(\mathrm{~s}, 3 \mathrm{H}), 3.86(\mathrm{~s}, 6 \mathrm{H}), 3.84(\mathrm{~s}, 6 \mathrm{H})$, $3.47(\mathrm{t}, J=7.1 \mathrm{~Hz}, 2 \mathrm{H}), 2.21 \sim 2.10(\mathrm{~m}, 2 \mathrm{H}) ;{ }^{13} \mathrm{C}$ NMR $\left(101 \mathrm{MHz}, \mathrm{CDCl}_{3}\right) \delta: 172.93,170.29,165.67,163.07(\mathrm{~d}$, $\left.J_{\mathrm{C}-\mathrm{F}}=12.0 \mathrm{~Hz}\right), 160.01,157.79,153.31,152.00,151.80$, $148.76\left(\mathrm{~d}, J_{\mathrm{C}-\mathrm{F}}=13.7 \mathrm{~Hz}\right), 139.40,138.94,125.62(\mathrm{~d}$, $\left.J_{\mathrm{C}-\mathrm{F}}=13.7 \mathrm{~Hz}\right), 124.97,120.09,116.54,116.29,111.77$,
111.56, 108.37, 104.87, 94.83, 91.41, 69.82, 59.98, 55.36, 54.80, 28.91, 25.43; ${ }^{19} \mathrm{~F}$ NMR (376 $\mathrm{MHz}, \mathrm{CDCl}_{3}$ ) $\delta$ : -102.73 (dd, $J=14.4,8.5 \mathrm{~Hz}$ ); HRMS calcd for $\mathrm{C}_{31} \mathrm{H}_{30^{-}}$ $\mathrm{O}_{8} \mathrm{~N}_{2} \mathrm{FS}[\mathrm{M}+\mathrm{H}]^{+}$609.1701, found 609.1685.

5,7-二甲氧基-3-(3-((6,7-二甲氧基喹唑啉-4-基)硫 基)丙氧基)-2-(3,4,5-三甲氧基苯基)-4 $\mathrm{H}$-色烯-4-酮 $\left(\mathbf{A}_{21}\right)$ : 白色固体, 收率 77.3\%. m.p. 193.3 194.5 ${ }^{\circ} \mathrm{C} ;{ }^{1} \mathrm{H}$ NMR $\left(400 \mathrm{MHz}, \mathrm{CDCl}_{3}\right) \delta: 8.72(\mathrm{~s}, 1 \mathrm{H}), 7.29(\mathrm{~s}, 2 \mathrm{H}), 7.16(\mathrm{~s}$, $1 \mathrm{H}), 7.11(\mathrm{~s}, 1 \mathrm{H}), 6.43(\mathrm{~d}, J=2.2 \mathrm{~Hz}, 1 \mathrm{H}), 6.29$ (d, $J=2.2$ $\mathrm{Hz}, 1 \mathrm{H}), 4.14$ (t, $J=6.1 \mathrm{~Hz}, 2 \mathrm{H}), 3.96(\mathrm{~d}, J=1.5 \mathrm{~Hz}, 6 \mathrm{H})$, 3.91 (s, 3H), 3.86 (s, 6H), 3.84 (s, 6H), 3.45 (t, $J=7.1 \mathrm{~Hz}$, 2H), $2.17(\mathrm{t}, J=13.1 \mathrm{~Hz}, 2 \mathrm{H}) ;{ }^{13} \mathrm{C}$ NMR $(101 \mathrm{MHz}$, $\left.\mathrm{CDCl}_{3}\right) \delta: 173.99,167.38,164.02,161.05,158.83,155.42$, $153.03,152.76,152.48,149.89,145.50,140.52,139.96$, $126.04,119.19,109.43,107.11,105.91,101.58,95.86$, $92.44,71.00,61.01,56.60 \sim 56.23,55.84,30.17,26.34$; HRMS calcd for $\mathrm{C}_{33} \mathrm{H}_{35} \mathrm{O}_{10} \mathrm{~N}_{2} \mathrm{~S}[\mathrm{M}+\mathrm{H}]^{+}$651.2007, found 651.2002 .

5,7-二甲氧基-3-(3-((7,8-二甲基喹唑啉-4-基)硫基) 丙氧基)-2-(3,4,5-三甲氧基苯基)- $4 \mathrm{H}$-色烯-4-酮 $\left(\mathbf{A}_{22}\right.$ ): 白 色固体, 收率 55.1\%. m.p. 150.1 151.2 ${ }^{\circ} \mathrm{C} ;{ }^{1} \mathrm{H}$ NMR $\left(400 \mathrm{MHz}, \mathrm{CDCl}_{3}\right) \delta: 8.85(\mathrm{~s}, 1 \mathrm{H}), 7.72(\mathrm{~d}, J=8.5 \mathrm{~Hz}$, $1 \mathrm{H}), 7.29 \sim 7.25(\mathrm{~m}, 3 \mathrm{H}), 6.43(\mathrm{~d}, J=2.2 \mathrm{~Hz}, 1 \mathrm{H}), 6.28(\mathrm{~d}$, $J=2.2 \mathrm{~Hz}, 1 \mathrm{H}), 4.13(\mathrm{t}, J=6.1 \mathrm{~Hz}, 2 \mathrm{H}), 3.90(\mathrm{~s}, 3 \mathrm{H}), 3.85$ (s, 6H), 3.83 (d, $J=2.2 \mathrm{~Hz}, 6 \mathrm{H}), 3.42(\mathrm{t}, J=7.2 \mathrm{~Hz}, 2 \mathrm{H})$, 2.58 (s, 3H), 2.41 (s, 3H), $2.20 \sim 2.11(\mathrm{~m}, 2 \mathrm{H}) ;{ }^{13} \mathrm{C}$ NMR $\left(101 \mathrm{MHz}, \mathrm{CDCl}_{3}\right) \delta: 173.96,170.92,164.01,161.05$, $158.82,153.03,152.75,152.55,146.84,141.96,140.52$, $139.96,134.31,129.64,126.05,122.26,120.70,109.44$, 105.91, 95.84, 92.44, 71.04, 61.01, 56.39, 55.83, 30.04, 26.29, 20.94, 13.07; HRMS calcd for $\mathrm{C}_{33} \mathrm{H}_{35} \mathrm{O}_{8} \mathrm{~N}_{2} \mathrm{~S}[\mathrm{M}+$ $\mathrm{H}]^{+}$619.2109, found 619.2101.

\subsection{2 目标化合物的体外抑菌活性测试}

采用浊度法测试目标化合物在 100 和 $50 \mu \mathrm{g} / \mathrm{mL}$ 浓 度下, 对水稻白叶枯病菌、柑橘溃疡病菌和烟草青枯病 菌三种植物病菌的体外抑制活性, 并对活性较好的化合 物进行 $\mathrm{EC}_{50}$ 值测试 ${ }^{[28]}$.

营养肉汤培养基(NB): $5 \mathrm{~g}$ 蛋白胨、 $1 \mathrm{~g}$ 酵母粉、 $10 \mathrm{~g}$ 葡萄糖、 $3 \mathrm{~g}$ 牛肉膏和 $1000 \mathrm{~mL}$ 蒸馏水, 并用质量分数 为 $20 \% \mathrm{NaOH}$ 溶液调节 $\mathrm{pH}$ 至 $7.2 \pm 0.2$; 营养琼脂培养 基(NA): $200 \mathrm{~mL} \mathrm{NB}$ 液体培养基中加入 $3.6 \mathrm{~g}$ 琼脂粉. 配 置好培养基后再用高压蒸汽灭菌锅 $\left(121{ }^{\circ} \mathrm{C}, 21 \mathrm{~min}\right)$ 进 行灭菌操作. 将灭菌好 NA 固体培养基趁热倒入无菌的 培养皿中, 待冷却后用平板划线法将三种测试菌进行接 种, 并在 $28{ }^{\circ} \mathrm{C}$ 恒温培养箱中活化; 用接种环将活化好 
的菌种放入到含有 NB 液体培养基的雉形瓶中, 并在恒 温摇床中振荡培养至对数生长期备用.

将目标化合物配制成 100 和 $50 \mu \mathrm{g} / \mathrm{mL}$ 浓度, 分别取 $1 \mathrm{~mL}$ 加入到装有 $4 \mathrm{~mL} \mathrm{NB}$ 液体培养基的试管中, 测定 每个浓度的加药无菌 NB 液体培养基的 OD 值, 然后加 入 $40 \mu \mathrm{L}$ 含有水稻白叶枯病菌、柑橘溃疡病菌和烟草青 枯病菌的 $\mathrm{NB}$ 液体培养基至试管中, 并在 $28{ }^{\circ} \mathrm{C} 、 180$ $\mathrm{r} / \mathrm{min}$ 恒温摇床培养, 待空白对照组 NB 液体培养基的 OD 值为 $0.6 \sim 0.8$, 用 96 孔板在酶标仪上测定各个浓度 的菌液的 $\mathrm{OD}$ 值.

校正 $\mathrm{OD}$ 值 $=$ 含菌培养基 $\mathrm{OD}$ 值 - 无菌培养基 $\mathrm{OD}$ 值 抑制率 $(\%)=($ 校正后对照培养基菌液 OD 值一校正后 含药培养基菌液 OD 值)/校正后对照培养基菌液 OD 值 $\times 100 \%$

\subsection{3 抗水稻白叶枯病菌活体测试}

采用剪叶法测试了目标化合物 $\mathbf{A}_{\mathbf{1 5}}$ 对水稻白叶枯病 菌的体内防治效果 ${ }^{[29]}$. 叶枯唑和噻菌铜为阳性对照. 阴 性对照(CK)为喷酒不含药液的二甲基亚砜(DMSO)水溶 液.

对水稻白叶枯病菌的体内保护活性: 先分别配制浓 度为 $200 \mu \mathrm{g} / \mathrm{mL}$ 的 $\mathbf{A}_{15}$ 、叶枯唑和噻菌铜溶液 $30 \mathrm{~mL}$, 然 后将药液均匀的喷酒在水稻植株上, $24 \mathrm{~h}$ 后, 将剪刀放 在含有水稻白叶枯病菌的 NB 液体培养基浸泡, 然后用 浸泡后的剪刀对水稻进行剪叶处理, 并在温室(温度: $28{ }^{\circ} \mathrm{C}$; 湿度: $95 \%$ )中培养 $16 \mathrm{~d}$ 后观察.

对水稻白叶枯病菌的体内治疗活性: 将剪刀放在含 有水稻白叶枯病菌的 NB 液体培养基浸泡, 然后用浸泡 后的剪刀对水稻进行剪叶处理, $24 \mathrm{~h}$ 后, 配制浓度为 200 $\mu \mathrm{g} / \mathrm{mL}$ 的 $\mathbf{A}_{15}$ 、叶枯唑和噻菌铜溶液 $30 \mathrm{~mL}$, 然后将药液 均匀的喷酒在被剪叶处理的水稻植株上, 并在温室(温 度: $28{ }^{\circ} \mathrm{C}$; 湿度: 95\%)中培养 $16 \mathrm{~d}$ 后观察.

$$
\begin{aligned}
& \text { 病害指数 }=\frac{\sum(\text { 各级病叶数 } \times \text { 该病级值 })}{\text { 调查总叶数 } \times \text { 最高病级值 }} \times 100 \% \\
& \text { 防治效率 }(\%)=(C-T) / C \times 100 \%
\end{aligned}
$$

$C$ 为空白对照 $(\mathrm{CK})$ 的病情指数, $T$ 为处理组的病害指数

\subsection{4 扫描电镜成像实验}

量取 $1.5 \mathrm{~mL}$ 对数生长期的烟草青枯病菌 (R. solanacearum) 和水稻白叶枯病菌 (X. oryzae) 的菌液于 $2 \mathrm{~mL}$ 离心管中, 低速离心 $(7000 \mathrm{r} / \mathrm{min}) 1 \mathrm{~min}$, 倒出上清液, 并加入 $1 \mathrm{~mL}$ 磷酸盐缓冲液(PBS)以洗去培养基, 重复三 次此过程. 再加入 $1.5 \mathrm{~mL}$ PBS 缓冲溶液, 摇晃使菌分 散, 再加入目标化合物 $\mathbf{A}_{\mathbf{3}}$ 和 $\mathbf{A}_{\mathbf{1 5}}$ 的药液使其浓度分别为 50 和 $100 \mu \mathrm{g} / \mathrm{mL}$, 空白对照补加二甲基亚砜(DMSO) 3 $\mu \mathrm{L}$, 并在 $28{ }^{\circ} \mathrm{C} 、 180 \mathrm{r} / \mathrm{min}$ 的恒温摇床作用 $10 \mathrm{~h}^{[30]}$.
作用完毕后，在低速离心(7000 r/min)约 $1 \mathrm{~min}$, 弃 去上清液, 菌体用 $1 \mathrm{~mL}$ PBS 溶液洗涤 3 次以洗去药剂; 加入 $1 \mathrm{~mL}$ 质量分数为 $2.5 \%$ 龙二醛固定液固定过夜 $\left(4{ }^{\circ} \mathrm{C}\right)$, 弃去固定液, 再用 $1 \mathrm{~mL}$ PBS 溶液洗涤 3 次以洗 去戊二醛; 菌体分别用 $1 \mathrm{~mL}$ 体积分数为 $30 \% 、 50 \%$ 、 $70 \% 、 90 \%$ 和 $100 \%$ 乙醇依次进行脱水置换(每次置换约 $10 \mathrm{~min}$ ), 脱水后样品置于冷冻干燥机冷冻干燥 $2 \mathrm{~h}$; 干 燥菌体粉末经粘样、喷金后进行 SEM 测试.

辅助材料(Supporting Information) 目标化合物 $\mathbf{A}_{\mathbf{1}} \sim$ $\mathbf{A}_{22}$ 的 ${ }^{1} \mathrm{H}$ NMR, ${ }^{13} \mathrm{C}$ NMR, HRMS 和部分化合物的 ${ }^{19} \mathrm{~F}$ NMR 的谱图. 这些材料可以免费从本刊网站(http:// sioc- journal.cn/)上下载.

\section{References}

[1] Fan, Z. J.; Shi, J., Luo, N.; Ding, M. H.; Bao, X. P. J. Agric. Food Chem. 2019, 67, 11598.

[2] Jiang, S. C.; Tang, X.; Chen, M.; He, J.; Su, S. J.; Liu, L. W.; Xue, W. Pest Manage. Sci. 2019, 76, 853.

[3] Tang, X.; Zhang, C.; Chen, M.; Xue, Y. N.; Liu, T. T.; Xue, W. New J. Chem. 2020, 44, 2374.

[4] Mo, F.; Ma, J.; Yang, X.; Zhang, P.; Li, Q.; Zhang, J. Phytomedicine 2020, 153223.

[5] Yu, M. S.; Lee, J.; Lee, J. M.; Kim, Y.; Chin, Y. W.; Jee, J. G.; Jeong, Y. J. Bioorg. Med. Chem. Lett. 2012, 22, 4049.

[6] Sun, F.; Zheng, X. Y.; Ye, J.; Wu, T. T.; Wang, J. L.; Chen, W. Nutr. Cancer 2012, 64, 599.

[7] Afroze, N.; Pramodh, S.; Hussain, A.; Waleed, M.; Vakharia, K. 3 Biotech 2020, 10, 211.

[8] Wang, S. J.; Tong, Y.; Lu, S.; Yang, R.; Liao, X.; Xu, Y. F.; Li, X. Planta Med. 2010, 76, 1492.

[9] Jiang, M.; Zhu, M.; Wang, L.; Yu, S. Biomed. Pharmacother. 2019 , 120, 109506.

[10] Guitard, R.; Paul, J. F.; Véronique, N. R.; Aubry, J. M. Food Chem. 2016, 213.

[11] Jiang, S. C.; Su, S. J.; Chen, M.; Peng, F.; Zhou, Q.; Liu, L. W.; Xue, W. J. Agric. Food Chem. 2020, 68, 5641.

[12] Li, P.; Chen, Y.; Xia, R. J.; Guo, T.; Zhang, M.; Jiang, S. C.; Tang, X.; He, M.; Xue, W. Chem. J. Chin. Univ. 2019, 40, 909 (in Chinese).

(李普, 陈英, 夏榕娇, 郭涛, 张敏, 蒋仕春, 汤旭, 贺鸣, 薛伟, 高等学校化学学报, 2019, 40, 909.)

[13] Liu, H. B.; Lv, P.; Pan, N. N.; Ai, L. M.; Liu, Y. X. Chem. J. Chin. Univ. 2014, 35, 981 (in Chinese).

(刘海涁, 吕萍, 潘宁宁, 艾丽梅, 刘永祥, 高等学校化学学报, 2014, 35, 981.)

[14] Xu, Y. Y. M.S. Thesis, Suzhou University, Suzhou, 2014 (in Chinese). (许芸芸，硕士论文，苏州大学，苏州, 2014.)

[15] Zhang, G. Q.; Guo, Q. Q.; Zhao, T. Y.; Wang, M.; Xing, L. N. Chin. J. Synth. Chem. 2020, 28, 491 (in Chinese). (张贵强, 郭晴晴, 易君明, 赵廷渊, 王梅, 邢林娜, 合成化学, 2020, 28, 491.)

[16] Dos, R.; Sharon, V.; Ribeiro, N. S.; Rocha, D. A.; Fortes, I. S.; Silva, T. D.; Andrade, S. F.; Macedo, A. J. Chem. Biol. Drug Des. 2020.

[17] Wan, Z. H. M.S. Thesis, Guizhou University, Guiyang, 2015 (in Chinese). (万治华, 硕士论文, 贵州大学, 贵阳, 2015.)

[18] Sun, H. Y.; Sun, H. S.; Liu, M. Z.; Huang, W.; Yang, G. F. Chin. J. Org. Chem. 2018, 38, 2067 (in Chinese). 
(孙海燕, 孙宏顺, 刘明珍, 黄伟, 杨光富, 有机化学, 2018, 38, 2067.)

[19] Wu, J.; Bai, S.; Yue, M.; Luo, L. J.; Shi, Q. C.; Ma, J.; Du, X. L.; Kang, S. H.; Hu, D. Y.; Yang, S. Chem. Pap. 2014, 68, 969.

[20] Gao, X.W.; Cai, X. J.; Yan, K.; Gao, L. L.; Wang, H. Y.; Chen, Z.; Song, B. A. Chin. J. Org. Chem. 2008, 28, 1785 (in Chinese). (高兴文, 蔡学建, 严凯, 高丽丽, 王和英, 陈卓, 宋宝安, 有机 化学, 2008, 28, 1785.)

[21] Li, Q. M.S. Thesis, Guizhou University, Guiyang, 2018 (in Chinese).

(李琴，硕士论文，贵州大学，贵阳, 2018.)

[22] Liu, J.H.; Liu, Y.; Jian, J. Y.; Bao, X. P. Chin. J. Org. Chem. 2013, 33, 370 (in Chinese). (刘军虎, 刘勇, 寒军友, 鲍小平, 有机化学, 2013, 33, 370.)

[23] Zhang, C. R.; Wang, L.; Ge, Y. L.; Ju, X. L. Chin. J. Org. Chem. 2007, 27, 1432 (in Chinese).

(张成仁, 王柳, 葛燕丽, 巨修练, 有机化学, 2007, 27, 1432.)

[24] Yang, Y.; Ma, Y. N.; Huang, T.; Xie, W.; Zhang, X.; Huang, G. S.; Liu, X. D. Chin. J. Org. Chem. 2018, 38, 2143 (in Chinese). (杨锐, 马艳妮, 黄婷, 解伟, 张霞, 黄国双, 刘小东, 有机化学, $\mathbf{2 0 1 8}, 38,2143$.

[25] Xue, W.; Song, B. A.; Zhao, H. J; Qi, X. B.; Huang, Y. J.; Liu, X. H. Eur. J. Med. Chem. 2015, 97, 155.

[26] Chen, Y.; Li, P.; Su, S. J.; Chen, M.; He, J.; Liu, L. W.; Xue, W. RSC Adv. 2019, 9, 23045.

[27] Long, C. W. M.S. Thesis, Guizhou University, Guiyang, 2015 (in Chinese).

(龙承文，硕士论文，贵州大学，贵阳，2015.)

[28] Zhang, C.; Jiang, S. C.; Chen, Y.; Guo, T.; Xia, R. J.; Tang, X.; Chen, L. J.; He, M.; Xue, W. Chin. J. Org. Chem. 2019, 39, 1160 (in Chinese).

(张橙，蒋仕春，陈英，郭涛，夏榕娇，汤旭，陈丽娟，贺鸣，薛 伟, 有机化学, 2019, 39, 1160.)

[29] Wang, L.; Zhou, X.; Lu, H.; Mu, X. F.; Jin, L. H. Molecules 2020. $25,1488$.

[30] Chen, Y.; Li, P.; Chen, M.; Su, S. J.; He, J.; Zhang, M.; Liu, L. W.; Xue, W. Chin. J. Org. Chem. 2019, 39, 2813 (in Chinese).

(陈英, 李普, 陈梅, 苏时军, 贺军, 张敏, 柳立伟, 薛伟, 有机 化学, 2019, 39, 2813.) 